\title{
Subcritical transition scenarios via linear and nonlinear localized optimal perturbations in plane Poiseuille flow
}

\author{
Mirko Farano ${ }^{1,2}$, Stefania Cherubini ${ }^{2}$, \\ Jean-Christophe Robinet ${ }^{2}$ and Pietro De Palma ${ }^{1}$ \\ ${ }^{1}$ DMMM, Politecnico di Bari, Via Re David 200, 70125 Bari, Italy \\ ${ }^{2}$ DynFluid Laboratory, Arts et Metiers ParisTech, 151 Boulevard de l'Hopital, 75013 \\ Paris, France \\ E-mail: m.farano@libero.it
}

\begin{abstract}
Subcritical transition in plane Poiseuille flow is investigated by means of a Lagrange-multiplier direct-adjoint optimization procedure with the aim of finding localized three-dimensional perturbations optimally growing in a given time interval (target time). Space localization of these optimal perturbations (OPs) is achieved by choosing as objective function either a p-norm (with $p \gg 1)$ of the perturbation energy density in a linear framework; or the classical (1-norm) perturbation energy, including nonlinear effects. This work aims at analyzing the structure of linear and nonlinear localized OPs for Poiseuille flow, and comparing their transition thresholds and scenarios. The nonlinear optimization approach provides three types of solutions: a weakly nonlinear, a hairpin-like and a highly nonlinear optimal perturbation, depending on the value of the initial energy and the target time. The former shows localization only in the wall-normal direction, whereas the latter appears much more localized and breaks the spanwise symmetry found at lower target times. Both solutions show spanwise inclined vortices and large values of the streamwise component of velocity already at the initial time. On the other hand, p-norm optimal perturbations, although being strongly localized in space, keep a shape similar to linear 1-norm optimal perturbations, showing streamwise-aligned vortices characterized by low values of the streamwise velocity component. When used for initializing direct numerical simulations, in most of the cases nonlinear OPs provide the most efficient route to transition in terms of time to transition and initial energy, even when they are less localized in space than the p-norm OP. The p-norm OP follows a
\end{abstract}


transition path similar to the oblique transition scenario, with slightly oscillating streaks which saturate and eventually experience secondary instability. On the other hand, the nonlinear OP rapidly forms large-amplitude bent streaks and skips the phases of streak saturation, providing a contemporary growth of all of the velocity components due to strong nonlinear coupling.

Keywords: conditional stability, nonlinear optimal, transition to turbulence, channel flow

\section{Introduction}

Transition to turbulence in shear flows is a longstanding problem that has been challenging generations of researchers since the early experiments of Reynolds (1883). It is now clear that, even for simple shear flows, transition cannot be linked only to a linear instability arising at a well-defined critical value of the Reynolds number, $R e_{c}$ (unconditional instability). For instance, pipe and plane Couette flows have an infinite value of $R e_{c}$ but can experience transition for sufficiently high values of the Reynolds number, $R e>R e_{g}$ (Bottin and Chaté 1998, Avila et al 2011). For other shear flows, such as plane Poiseuille and boundarylayer flows, even if a finite value of $R e_{c}$ does exist, transition is often subcritical, being observed at $R e_{g}<R e<R e_{c}$ when the laminar base flow is perturbed with a sufficiently high amplitude (see Patel and Head (1969), Schlichting and Gersten (2000)). In these conditions the stability of the flow is conditional, namely it depends on the shape and intensity of perturbations brought to the base flow. The determination of the minimum-energy perturbation capable of leading the flow to transition is a crucial issue for understanding subcritical transition and indeed is a difficult task. In fact, shear flows can be very sensitive to small modifications of the initial perturbations, as proven in the experiment by Darbyshire and Mullin (1995), in which the authors observed that the same perturbation (within the accuracy of the experimental resolution) may lead to very different outcomes in terms of transition to turbulence.

With the aim of determining transition thresholds, in the last decades there has been a large interest in perturbations transiently amplifying due to the non-normality of the NavierStokes operator. These small-amplitude perturbations can achieve in a finite time large amplitudes, sufficient to trigger nonlinear effects leading the flow to transition (Trefethen et al 1993, Landahl 1980). The search for these transiently growing perturbations led to the concept of 'optimal perturbations' experiencing the largest amplification over a finite time horizon. For plane channel flow, Butler and Farrell (1992) found that, in a linear framework, the maximum energy amplification over time can be obtained by a pair of streamwiseinvariant vortices generating at a finite time a pair of streamwise-invariant streaks (Farrell 1988). However, these streamwise-invariant structures are strongly different from perturbations that are usually observed in real flows during transition. In fact, in real flow configurations, disturbances are mostly due to localized imperfections of the wall surface (for pipes and channels), or to freestream turbulence and acoustic waves (in the case of boundarylayer flows). As a result, transition to turbulence is often observed in localized spots or puffs (Wygnanski and Champagne 1973, Wygnanski et al 1976, Perry et al 1981, Singer 1996) which spread in space and merge, leading the flow to sustained turbulence. The early phases of development of turbulent spots or puffs originated by localized perturbations (Perry 
et al 1981, Singer 1996), localized pulses or jets of finite amplitude have been used to explain the intrinsic mechanisms of subcritical transition to turbulence in pipe flow (Hof et al 2005, Mellibovsky and Meseguer 2007), and in plane Couette flow (Dauchot and Daviaud 1995). The formation of turbulent spots from localized perturbations for $R e_{g}<R e<R e_{c}$ has been investigated in channel and boundary layer flows (Henningson et al 1993, Levin and Henningson 2007). However, in these studies the chosen initial perturbations have simple shapes such as pulses, jets (Singer and Joslin 1994), localized pairs of alternated vortices (Levin and Henningson 2007) or rings (Suponitsky et al 2005). Those perturbations cannot guarantee a large energy growth such as optimal perturbations; thus, they need very large initial amplitudes to achieve transition, largely outgrowing the transition thresholds sought for the considered flows. Thus, in order to accurately evaluate such energy thresholds for more realistic, localized perturbations, an energy optimisation inducing the localization of perturbations would be more appropriate (Cherubini et al 2010, Monokrousos et al 2010).

Recently, constrained optimisations of the p-norm of the disturbance energy have been used by Foures et al (2013), with the aim of promoting localization of the optimal perturbation. Using higher-order norms as objective function allows one to reduce the spatial extent of the optimal perturbation (Foures et al 2013), revealing the existence of possible 'hotspots' in the flow. This technique can be very useful for designing experimental setups, in which perturbations can be injected only locally, as well as control devices, in which a 'minimalenergy' actuation is desired. Increasing the order of the energy norm in the objective function, the resulting optimal disturbance becomes more and more localized even for simple shear flows such as plane Poiseuille flow; whereas, for these parallel shear flows, a linear optimisation of the $L_{2}$-norm of the perturbation velocity vector always leads to spatially extended structures such as streamwise-invariant streaks. For plane Poiseuille flow, linear p-norm optimizations performed by Foures et al (2013) in a two-dimensional framework have provided two types of localized optimal disturbances: one localized at the center of the channel and the other one at the wall, capable to extract energy from the base flow in two different ways. However, how p-norms behave in a three-dimensional flow configuration, in the presence of a strong transient non-local mechanism such as the lift-up effect (Landahl 1980) has not yet been investigated. In particular, it has still not been established whether those localized linear optimals are indeed efficient in inducing subcritical transition, in terms of both time to transition and initial energy.

Recent studies have shown that, for simple shear flows, transition thresholds can be accurately determined by using energy optimisations including nonlinear effects (Rabin et al 2012, Cherubini et al 2015, Duguet et al 2013). By this approach, the optimization procedure naturally leads to large-amplitude localized disturbances capable of producing a large energy growth due to nonlinear local effects. Nonlinear optimizations have been performed for pipe (Pringle and Kerswell 2010, Pringle et al 2012), boundary layer (Cherubini et al 2010, 2011, 2015) and Couette (Monokrousos et al 2011, Rabin et al 2012, Cherubini et al 2013, Duguet et al 2013) flows. These authors optimize a functional linked to the turbulent dynamics, namely the perturbation kinetic energy (see Pringle and Kerswell (2010), Pringle et al (2012), Rabin et al (2012), Cherubini and De Palma (2013), Duguet et al (2013)) or the time-averaged dissipation (see Monokrousos et al (2011)), including nonlinear terms into the optimization and thus following the evolution of the perturbation until transition is initiated. Optimizing for long times, and bisecting the initial energy, one can find the perturbation of minimal energy which brings the flow on the verge of transition (Rabin et al 2012, Duguet et al 2013, Cherubini et al 2015). In fact, the amplification of such nonlinear optimal disturbances largely outgrows that of the linear optimals (Cherubini et al 2011, Pringle et al 2012), leading to transition for lower values of the initial energy. 
However, it is still not clear how this large energy growth is linked to the shape and/or to the higher amplitude of the nonlinear perturbations. When comparing a linear (non-localized) and a nonlinear (localized) optimal perturbation having the same initial energy, not only the shape of the two optimals differs, but also their relative amplitudes, since the localization property of the latter induces larger velocity values for the same energy. A more appropriate comparison should make use of localized linear optimal perturbations, such as those obtained by a linear p-norm optimization (Foures et al 2012).

The present work aims at analyzing and comparing the structure and transition scenarios of localized linear and nonlinear optimal perturbations in the plane Poiseuille flow at subcritical values of the Reynolds number. The comparison of the different outcomes, in terms of energy growth and consequent transition, between the linear p-norm and the nonlinear energy optimization, allows us to analyze: (i) the effects of nonlinearity in the localization of perturbations; (ii) the relative importance of the shape and amplitude of different initial perturbations for inducing transition.

The paper is organized as follows. In the second section we define the problem and describe the optimization methods. In the third section, divided into two parts, a thorough discussion of the results of the two optimization approaches is provided. In particular, in the first part, the focus is on the comparison of the shape and amplitudes of the two different types of optimal perturbations at different target times and initial energies. Whereas, the second part unravels the route to transition of both optimal perturbations, and their relative effectiveness in terms of initial energy and time to transition. Finally, some concluding remarks are provided.

\section{Problem formulation}

As a suitable test for our energy-growth analysis, we consider the incompressible flow driven by a constant pressure gradient between two plates, known as the plane Poiseuille flow. Nondimensional variables are chosen such that half the distance between the plates is $h=1$ and the centerline velocity of the laminar flow is $U_{c}=1$. The dynamics of such a flow is governed by the Navier-Stokes (NS) equations:

$$
\begin{aligned}
& \frac{\partial U_{i}}{\partial x_{i}}=0 \\
& \frac{\partial U_{k}}{\partial t}+\frac{\partial\left(U_{k} U_{j}\right)}{\partial x_{j}}+\frac{\partial P}{\partial x_{k}}-\frac{1}{R e} \frac{\partial^{2} U_{k}}{\partial x_{j}^{2}}=0
\end{aligned}
$$

where $t$ is the time, $R e$ is the Reynolds number, $P$ is the pressure, $\mathbf{U}$ is the velocity vector having components $U, V, W, \boldsymbol{x}$ is the position vector in space having components $x, y, z: x$ for the streamwise direction, $y$ for the wall-normal direction, and $z$ for the spanwise direction. With the aforementioned normalization, the laminar-flow solution is given by the following parabolic profile:

$$
\bar{U}=y(2-y) ; \quad \bar{V}=0 ; \quad \bar{W}=0 .
$$

This velocity profile is considered as the base flow, $\left(\overline{U_{i}}, \bar{P}\right)$, onto which finite amplitude perturbations, $\left(u_{i}, p\right)$, are superposed. The evolution of these perturbations is governed by the Navier-Stokes equations written in perturbative formulation, which are solved imposing Dirichlet boundary conditions for the three velocity components at the $y$-constant boundaries, whereas periodicity of the perturbation is prescribed in the spanwise and streamwise 
directions. All computations have been performed with $R e=4000$. If not specified, the streamwise and wall-normal dimensions of the domain are equal to $2 \pi$ and 2 , respectively, whereas the spanwise domain length is equal to $\pi$. Notice that, for validation purposes, we have chosen the same Reynolds number and the same streamwise and wall-normal domain dimensions employed by Foures et al (2013) in a two-dimensional framework. The chosen domain is discretized using a staggered grid with $300 \times 100 \times 120$ points and the NavierStokes equations are solved by a fractional-step method with second-order accuracy in space and time (Verzicco and Orlandi (1996)).

\subsection{The optimization problem}

The optimization procedure employed in the present analysis aims at computing the velocity perturbation at $t=0$ providing the maximum value of the objective function at a given target time, $T_{\text {opt }}$. The chosen objective function is the ratio between the p-norm of the energy density, $E_{p}$, measured at target time $T_{\text {opt }}$, and the initial energy $E(0)$, where:

$$
E_{p}(t)=\left(\frac{1}{V} \int_{V}(\mathrm{e}(t))^{p} \mathrm{~d} V\right)^{1 / p}=\left(\frac{1}{V} \int_{V}\left(\frac{1}{2} u_{i} u_{i}(t)\right)^{p} \mathrm{~d} V\right)^{1 / p}
$$

is the p-norm of the energy density and

$$
E(0)=\frac{1}{V} \int_{V} \frac{1}{2} u_{i} u_{i}(0) \mathrm{d} V
$$

is the initial energy.

For $p=1$, the classical definition of the energy density (often called 1-norm in this work) is recovered. As discussed by Foures et al (2013), increasing the value of $p$, the optimal perturbation will be more and more localized in a limited region of the domain with a higher energy density. It is worth noticing that a similar localization effect has been recovered in previous works based on a nonlinear optimization procedure, using the 1-norm of the energy density as objective function (Cherubini et al 2011, Pringle et al 2012, Monokrousos et al 2011).

The optimization problem is subject to partial differential constraints (Hinze and Pinnau (2009)), namely the perturbative NS equations. For nonlinear optimization, the initial value of the energy density is imposed $\left(E(0)=E_{0}\right)$. This choice is motivated by the possibility of comparing the results with the 1-norm optimization having the same initial energy. In order to optimize the chosen objective function subject to these constraints, a Lagrange multiplier technique is used (see Zuccher et al (2004)). The method consists of adding these constraints to the objective function via scalar product with the Lagrange multipliers (or adjoint variables) $\left(u_{i}^{\dagger}, p^{\dagger}, \lambda\right)$, yielding an augmented functional provided in the appendix. This functional is then derived with respect to the direct and adjoint variables, leading to the adjoint equations, as well as the compatibility conditions, which are responsible for the localization of the optimal perturbation when the p-norm of the energy density is used as objective function (see the discussion in the appendix). These equations are solved by using an iterative procedure based on the successive integration of the direct and adjoint equations, coupled with a gradient-based methods as discussed in more detail in the appendix. Convergence is attained when the variation of the objective function between two successive iterations is smaller than a chosen threshold, equal to $\epsilon=10^{-5}$. 


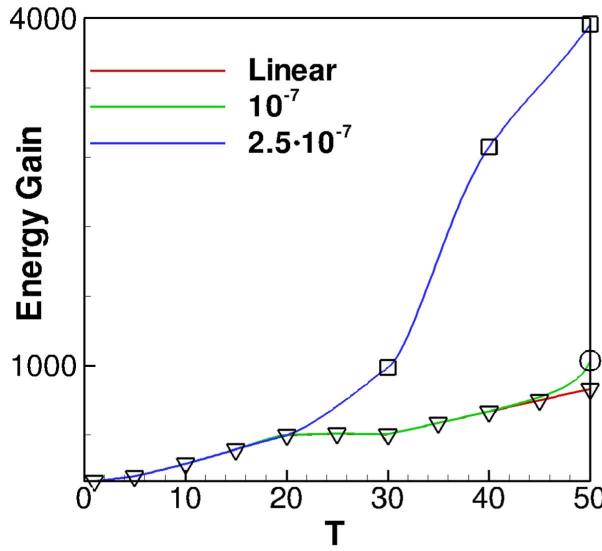

(a)

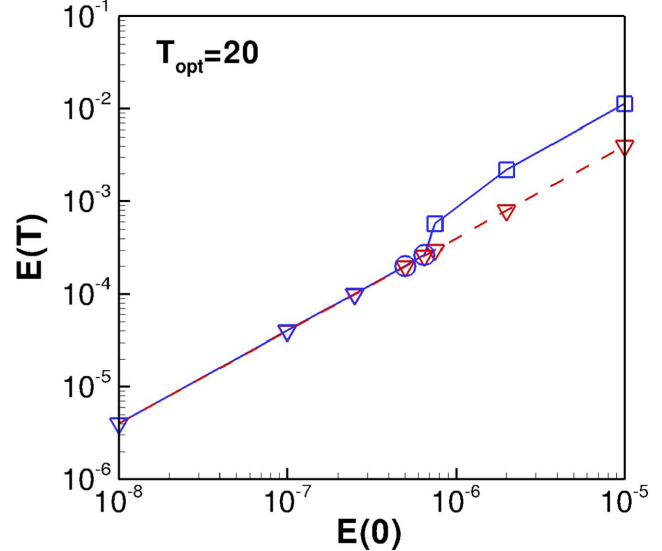

(b)

Figure 1. (a) Optimal energy gain curve versus target time obtained with linear (red line) and nonlinear 1-norm optimization with initial energy $E_{0}=10^{-7}$ (green line) and $E_{0}=2.5 \times 10^{-7}$ (blue line). (b) Optimal energy at target time versus initial energy for $T_{\mathrm{opt}}=20$ for a nonlinear (solid line) and a linear (solid line) 1-norm optimization. The different shapes of the symbols indicate different types of optimal solutions resulting from the optimisation at the chosen $T_{\mathrm{opt}}$ and $E_{0}$ : triangles for linear optimals, circles for weakly nonlinear and squares for highly nonlinear optimals.

\section{Results}

The linear and nonlinear optimization procedures described above have been employed to study subcritical transition in plane Poiseuille flow at $R e=4000$. Linear and nonlinear optimal perturbations with $p=1$ will be presented in section 3.1.1. Then, in section 3.1.2, the value of $p$ will be increased, and the linear p-norm optimal perturbations will be discussed and compared with the previous ones.

To allow a meaningful comparison between linear and nonlinear results, the work will focus on rather small target times, $T_{\mathrm{opt}}<50$. However, as discussed in section 3.1.1, the results at the considered target times appear representative of the dynamics that can be found at larger times.

\subsection{Optimal perturbations}

3.1.1. Nonlinear 1-norm optimization. Nonlinear optimisations have been performed for several initial energies in the range $\left[10^{-8}, 10^{-5}\right]$, and for different target times, $T_{\mathrm{opt}}=10,15$, $16,18,20,30,40,50$ (for the largest ones, the value of the initial energy has been limited to $10^{-6}$ in order to avoid transition at the target time, allowing convergence of the optimization algorithm). Figure 1(a) provides the optimal energy gain computed for several target times by means of linear (red line) and nonlinear (green and blue lines) 1-norm optimizations using two different values of the initial energy. The three curves demonstrate the strong increase of the energy gain due to nonlinear effects, for sufficiently large target times and initial energies. The strong effect of the initial energy on the resulting energy gain can be also observed in figure 1(b), providing the energy at target time versus the initial energy obtained with a nonlinear optimisazion for $T_{\mathrm{opt}}=20$. The chosen values of $T_{\mathrm{opt}}$ and $E_{0}$ strongly influence also the shape of the resulting optimal perturbations; in particular, we have found four families of 

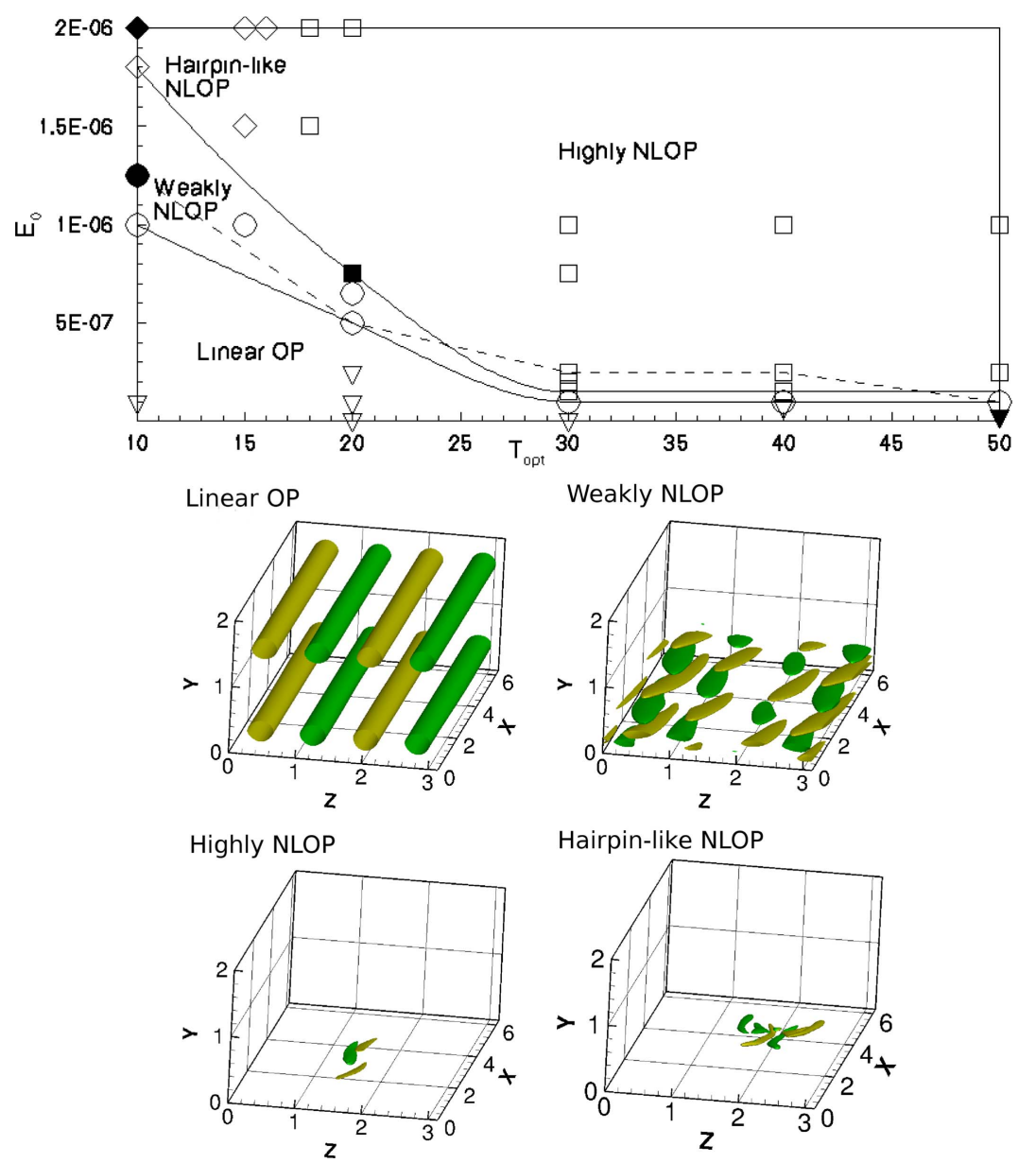

Figure 2. Different families of optimal perturbations on the plane $T_{\mathrm{opt}}$ and $E_{0}$. In the top frame, the symbols indicate the different types of optimal solutions resulting from the optimisation at the chosen $T_{\mathrm{opt}}$ and $E_{0}$ : triangles for linear optimals; circles for weakly nonlinear; squares for highly nonlinear optimals; and diamonds for hairpin-like optimals. The solid lines indicate the energy thresholds for shape modification. In the bottom frames, the linear, weakly nonlinear, highly nonlinear, and hairpin-like optimal solutions are represented using iso-surfaces of the Q-criterion, coloured in yellow/ green for positive/negative values of the streamwise vorticity, respectively. The optimal solutions shown are the ones represented by full symbols in the top frame. The dashed line represents the transition thresholds for the nonlinear optimals given in table 2 .

optimal perturbations, which are represented by different symbols in figures 1(a) and (b) as well as in figure 2. The different shapes of the four types of optimal perturbations are shown in the bottom subframes of figure 2 for comparison purposes, using the iso-surfaces of the Q-criterion $\left(Q=\frac{1}{2}\left(\|\Omega\|^{2}-\|S\|^{2}\right), \Omega\right.$ and $S$ being the vorticity tensor and the rate-of-strain tensor, respectively, according to Hunt et al (1988)). The first one (first subframe represented by triangles in the top frame) corresponds to the linear optimal perturbation (LOP), which is also obtained by nonlinear optimisations for sufficiently small values of $E(0)$. The second type, shown in the second subframe and represented by circles in the top frame of figure 2 , is 
labeled weakly nonlinear optimal perturbation (WNLOP), and it is found for increasing values of $E(0)$. This optimal solution shows a localization only in the wall-normal direction. Increasing the initial energy for $T_{\mathrm{opt}} \geqslant 20$ we have found optimal solutions of the third family, the highly nonlinear optimal perturbations (HNLOP), shown in the third subframe and represented by squares in the top frame of figure 2 . These solutions are localized in the three spatial directions and do not present any symmetry. Whereas, for large values of $E(0)$ and very small target times $\left(T_{\mathrm{opt}}<18\right.$ ), we obtain hairpin-like nonlinear optimal solutions (see also Farano et al (2015)), shown in the fourth subframe and represented by diamonds in the top frame of figure 2. The latter type of nonlinear optimal perturbation is associated with very large values of the initial energies, much larger than the transition threshold energy characterizing the other types of perturbations. Thus, their analysis is beyond the scope of this work since we are interested in comparing transition threshold between nonlinear 1-norm and linear p-norm optimal perturbations, thus employing energy levels close to this threshold. In the following we will thus analyze in detail the weakly and highly nonlinear optimal perturbations, whereas for further details about the hairpin-like optimal solution the reader is referred to Farano et al (2015). Moreover, figure 2 provides by solid lines the approximate threshold energy values for which the shape of the perturbation changes from one family to the other. As one can observe in figure 2, these energy thresholds vary strongly for a small target time, and converge towards a constant values for $T_{\mathrm{opt}} \geqslant 30$. This result has motivated our choice to limit $T_{\text {opt }}$ to a maximum value of 50. For comparison purposes, we have represented by the dashed line the threshold energy at which the computed nonlinear optimal perturbations are able to lead the flow to transition (see also the values in table 2 and the related comments in section 3.2). One can observe that, at the transition threshold, the OPs are either of weakly nonlinear or highly nonlinear type, depending on the target time, so that the transition threshold does not correspond to any of the shape-associated thresholds represented by the solid lines. Remarkably, linear OP are not found to induce transition for the initial energy at which they are obtained by the optimisation, even when noise is added to the initial perturbation field (see the discussion in section 3.2).

The weakly nonlinear optimal solution is shown at initial time in figure 3(a), for $T_{\text {opt }}=10, E_{0}=1.25 \times 10^{-6}$, and in figure $3(\mathrm{c})$ for $T_{\text {opt }}=20, E_{0}=6.5 \times 10^{-7}$. Such a solution shows a space localization in the wall-normal direction with respect to a linear optimal perturbation which instead occupies both the walls of the channel. One can observe that both initial optimal solutions shown in figures 3(a) and (c), as well as their evolution at target times (figures 3(b) and (d)) are localized on one of the two walls. Moreover, their shape is quite different from the linear optimal perturbations found by Butler and Farrell (1992), which are characterized by streamwise-aligned vortices. In fact, weakly nonlinear optimal solutions are characterized by alternated vortices inclined with respect to the streamwise direction, which lay on the flanks of a region of negative streamwise velocity disturbance. The vortices are inclined with respect to the mean flow, both in the wall-normal and in the spanwise direction. The upstream tilting with respect to the wall-normal direction is linked to the Orr mechanism (Schmid and Henningson (2001)) already observed in the linear case, whereas the spanwise tilting is not found in the linear case. However, the spanwise inclination of the initial vortices is a common feature of nonlinear optimal perturbations in shear flows, such as the ones found in the Blasius boundary-layer (Cherubini et al 2011), the Couette (Cherubini and De Palma 2013), and the asymptotic suction boundary layer flow (Cherubini et al 2015). One can also notice in figure 3 that, increasing the target time, the vortices elongate in the streamwise direction, without loosing their spanwise inclination. Thus, this first type of nonlinear optimal structure appears to break the wall-normal symmetry of the base flow, providing the first effects of localization induced by nonlinearity. 


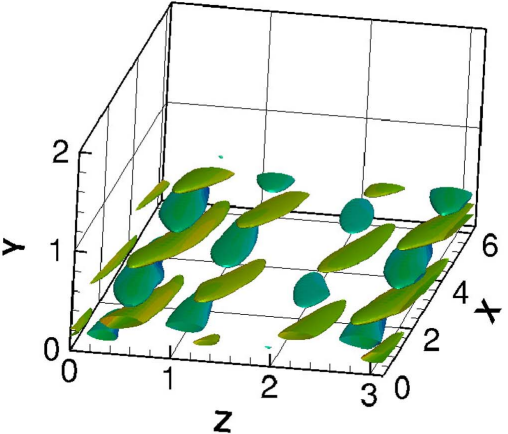

(a) $\mathrm{t}=0$

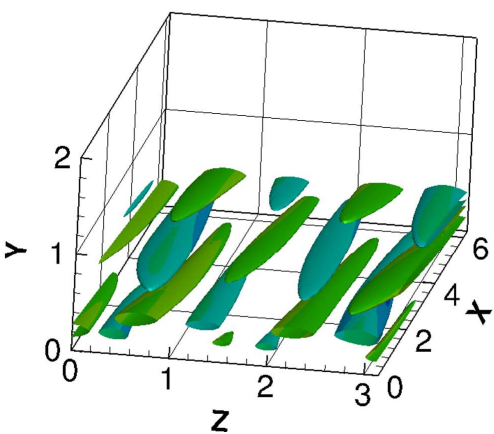

(c) $\mathrm{t}=0$

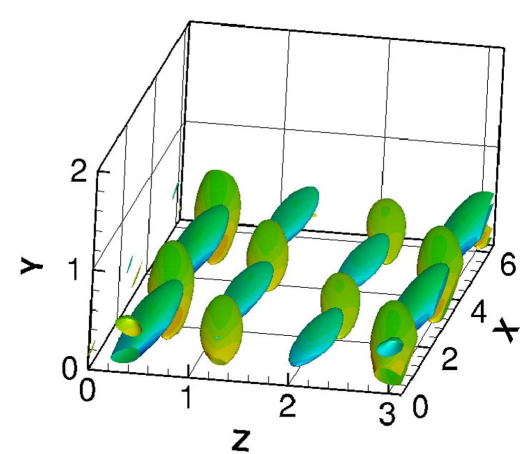

(b) $t=10$

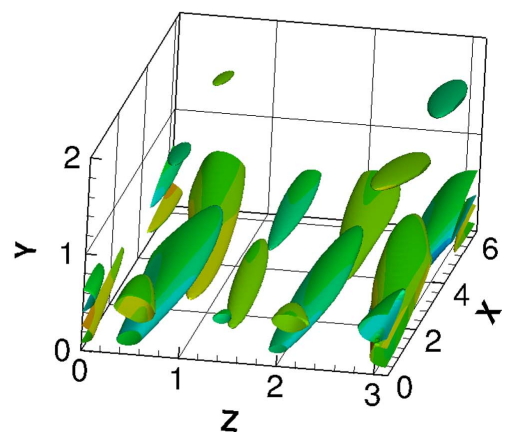

(d) $t=10$

Figure 3. Shape of the nonlinear 1-norm optimal perturbation (WNLOP solution) for (a) $T_{\mathrm{opt}}=10, E_{0}=1,25 \times 10^{-6}$ and (b) $T_{\mathrm{opt}}=20, E_{0}=6.5 \times 10^{-7}$. Isosurfaces of the Q-criterion at $t=0, Q=0.000195$ (a), $Q=0.00011$ (c) and (b), (d) at $t=T_{\text {opt }}(Q=0.011$ (b), $Q=0.003(\mathrm{~d}))$. The isosurfaces are colored by contours of streamwise vorticity.

As shown in figure 2, increasing the target time as well as $E_{0}$, the HNLOP is obtained. Figure 4 demonstrates that this optimal perturbation does not present any symmetry; it is characterized by localized staggered-inclined vortices on the flanks of packets of large streamwise velocity perturbation. Such a structure recalls the sinuous localized oscillation of streaks, as well as the nonlinear optimal solution also found for a Couette flow by Cherubini and De Palma (2013). This solution is quite different from the weakly nonlinear one, mostly for its strong spatial localization in the streamwise and spanwise directions. In fact, all of the velocity components decrease of two order of magnitude in $x$ and $z$ directions going from the perturbation towards the boundaries of the computational domain. Further increasing the initial energy and/or the target time, the optimal perturbation maintains a similar basic structure, as one can observe in figures 4 (c) and (d) for $T_{\mathrm{opt}}=30$. It is noteworthy that the same localized structure is found for a larger domain length, although it can be replicated several times in the spanwise or in the streamwise direction, for large initial energies.

3.1.2. Linear p-norm optimization. In the previous section we have discussed the progressive localization of the optimal perturbations when increasing the target time and the initial energy, for a nonlinear optimization of the energy gain. In this section we aim at investigating 


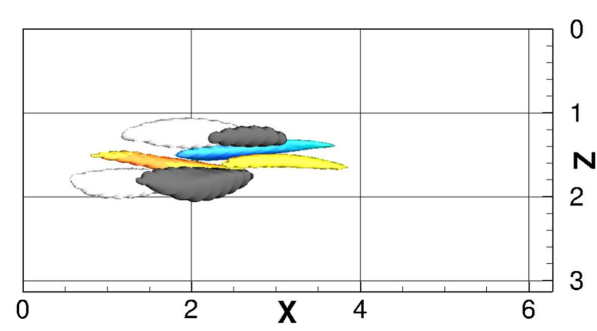

(a) $\mathrm{t}=0$

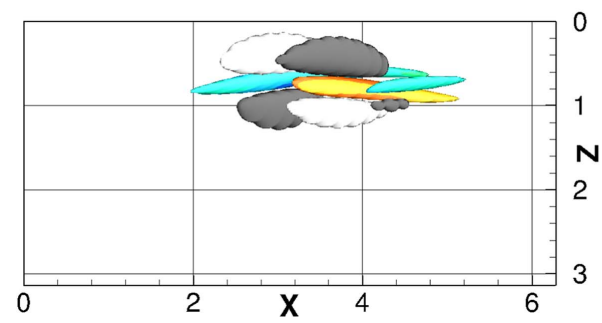

(c) $t=0$

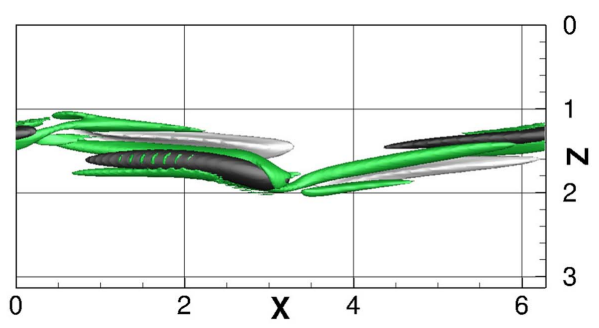

(b) $t=20$

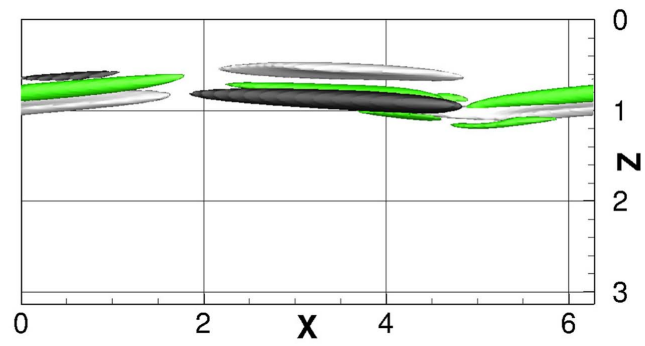

(d) $t=30$

Figure 4. Nonlinear 1-norm optimal perturbation (HNLOP solution) for (a), (b) $T_{\text {opt }}=20$ and $E_{0}=7,5 \times 10^{-7}$; (c), (d) $T_{\text {opt }}=30$ and $E_{0}=2.5 \times 10^{-7}$. Isosurfaces of the Q-criterion (colored) and of the streamwise velocity disturbance (light grey for positive and black for negative values) at (a) $t=0(Q=0.005, u= \pm 0.07)$, (b) $t=20$ $(Q=0.2, u= \pm 0.2),(\mathrm{c}) t=0(Q=0.002, u= \pm 0.04)$, and (d) $t=30(Q=0.1$, $u= \pm 0.15)$. The Q-criterion surfaces are colored by contours of the streamwise vorticity.

the effect of increasing the energy norm order, $p$, on the shape and amplitude of the optimal disturbance. We will discuss analogies and differences between the nonlinear 1-norm optimals and the linear p-norm optimal perturbations in order to uncover the basic mechanisms of energy amplification in the linear and nonlinear framework, for threedimensional Poiseuille flow.

Figure 5 provides the distribution of the energy gain and of the p-norm gain versus the value of $p$, for $T_{\mathrm{opt}}=10$. When $p$ is increased, the energy gain decreases (left frame), since it is not the objective function of the optimization process. On the other hand, the p-norm gain increases with $p$ (right frame), saturating for large values of the norm order. A similar behavior has been found also for larger target times, the p-norm gain converging toward a constant value for $p \rightarrow 50$. Thus, the largest value of $p$ used here, $p=50$, can be considered representative for maximizing the p-norm gain (as also concluded by Foures et al (2013) for the two-dimensional case).

In a two-dimensional framework, p-norm optimizations have been found very sensitive to the initialization of the optimisation procedure. By changing the initial guess, Foures et al (2013) found two different p-norm optimal solutions: one was localized at the center of the channel whereas the other was localized along one of the walls. Thus, following Foures et al (2013), we have used random noise perturbation localized in several zones of the domain to initialize the computations. In particular, the localization of the initial guess has been obtained by multiplying a perturbation field of white noise by the function $f\left(x_{i}\right)=\left(x_{i}-x_{i}^{+}\right)^{2}\left(x-x_{i}^{-}\right)^{2} \exp \left(\left(x_{i}-x_{i}^{c}\right)^{2} / \sigma\right)$ where $x_{i}^{+,-}$indicate the maximum/minimum value of the selected spatial coordinate, $x_{i}^{c}$ is the point at which we choose to localize the 


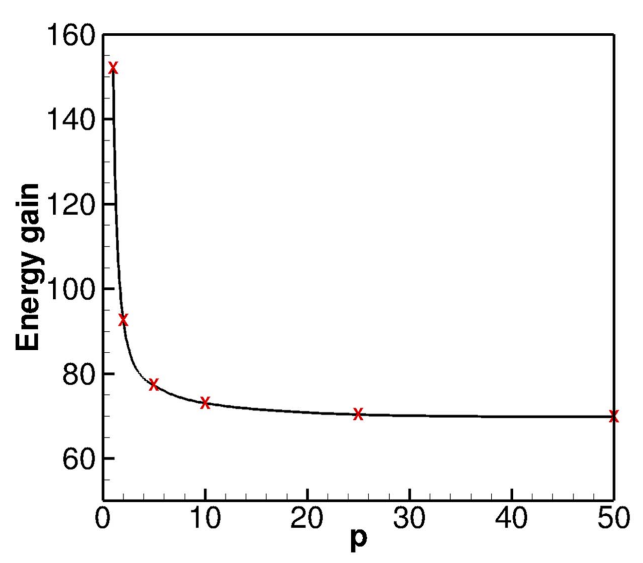

(a) Energy gain

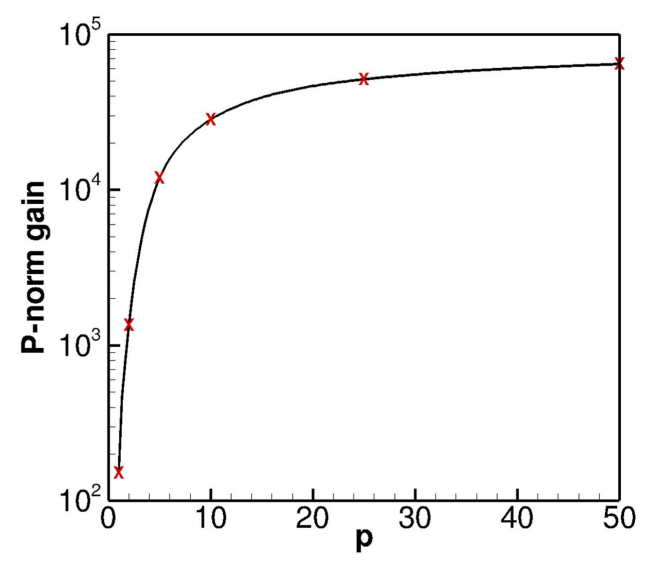

(b) P-norm gain

Figure 5. (a) Energy gain and (b) p-norm gain as a function of $p$ for $T_{\mathrm{opt}}=10$. Each symbol (x) represents an optimization.

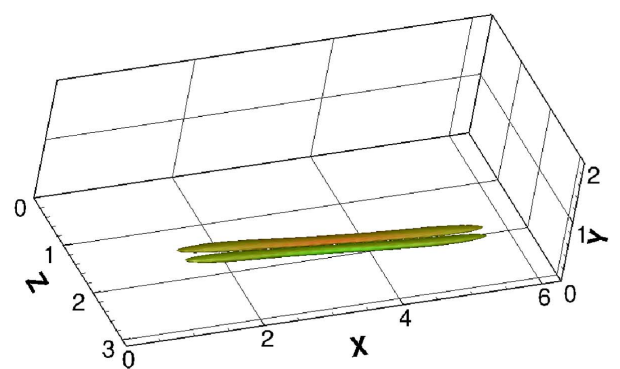

(a) $\mathrm{t}=0$

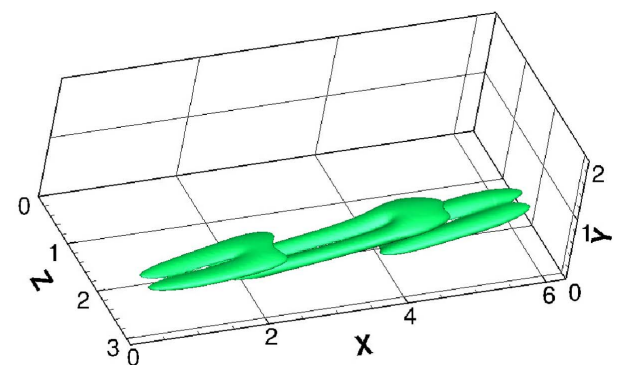

(b) $t=10$

Figure 6. 50-norm linear optimal perturbation for $T_{\mathrm{opt}}=10$. Isosurfaces of Q-criterion ( $Q=0.01$ ) at (a) $t=0$ and (b) $t=10$. The isosurfaces (a) are colored by contours of the stramwise vorticity.

perturbation, and $\sigma$ is its chosen length. This procedure has been validated by performing optimisations in a two-dimensional framework, reproducing exactly the two types of p-norm optimals found by Foures et al (2013). However, for three-dimensional perturbations, we have found only one type of solution, independent of the initialization of the optimization process. This optimal solution is also very robust with respect to changes of the parameter $p$. In fact, the same localized solution is found already for $p=2$, the only difference with respect to the one found for $p=50$ being the values of the velocity components. In all cases, the shape of the disturbance slightly varies as long as $p \geqslant 2$. This is probably due to the existence, in three space dimensions, of the lift-up mechanism, which is a very powerful mechanism dominating the perturbation growth.

The optimal 50-norm perturbation obtained for $T_{\mathrm{opt}}=10$ is shown in figure 6. It is characterized by a pair of vorticity tubes, highly localized at one wall and tilted upstream. These vorticity tubes do not show any inclination in the spanwise direction, unlike the highly nonlinear optimal solution; however, both solutions are characterized by very thin vortices tilted upstream. At target time, after tilting, the vortices connect each other, creating a 'tooth' 


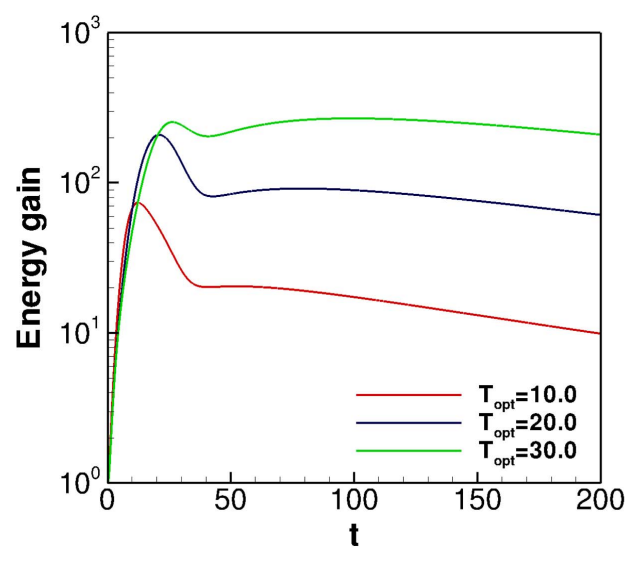

(a) Energy gain

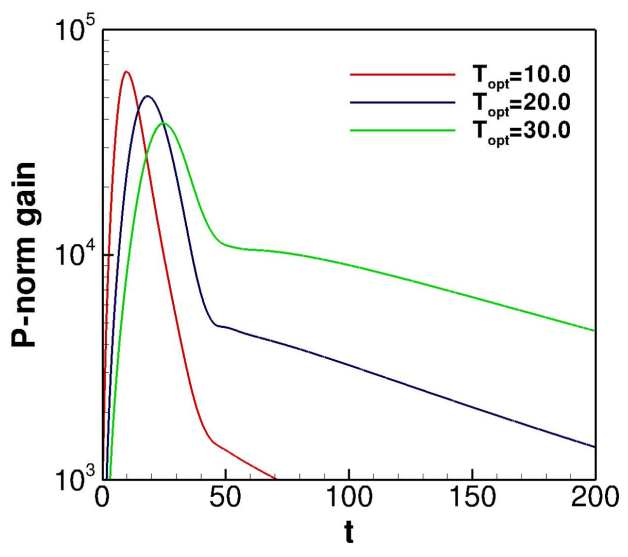

(b) P-norm gain

Figure 7. Evolution in time of the energy and 50-norm gain for the linear 50-norm optimal perturbation with three different optimization time.

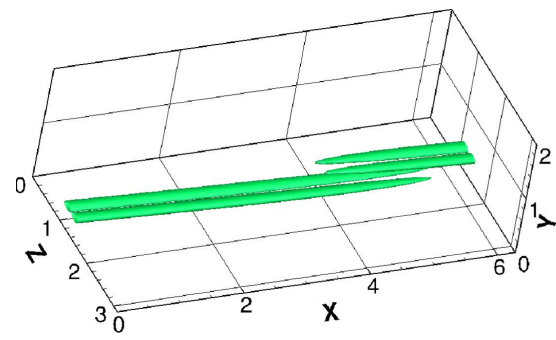

(a) $L_{x}=2 \pi$

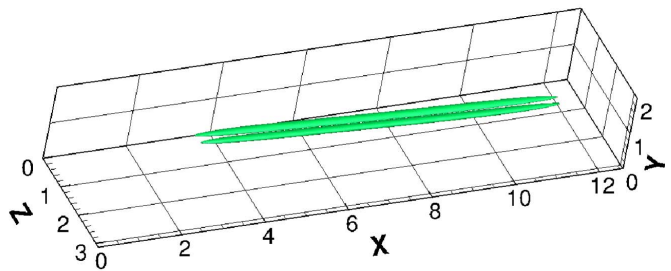

(b) $L_{x}=4 \pi$

Figure 8. 50-norm linear optimal perturbation for $T_{\text {opt }}=20$ and (a) $L_{x}=2 \pi$, and (b) $L_{x}=4 \pi$, at $t=0$. Isosurfaces of Q-criterion $(Q=0.01)$.

structure that resembles a 'linear' precursor of a hairpin vortex. However, since nonlinearity is necessary to sustain hairpin structures, at larger times this 'tooth' structure relaxes towards a pair of streamwise vortices inducing streamwise streaks, as it will be shown in the next section.

Increasing the target time, we obtain a larger energy gain, as one can observe in figure 7, providing the time evolution of the energy gain (a) and of the p-norm gain (b) for three different target times obtained using the linearized Navier-Stokes equations (compare with the values shown in figure 1 for the 1-norm linear and nonlinear case). Moreover, as shown in figure 8, for an increase of the target time the localized perturbation increases its streamwise length. This is due to the fact that the only energy growth mechanisms in a linear framework are the Orr and the lift-up effects: for the former to reach the maximum energy growth at a larger time, the structure must be longer to complete the tilting in a longer time (compare figure 8(a) with figure 6(a)). This elongation of the perturbation is linked to a decrease of the optimal p-norm gain, shown in figure 7(b), as well as to an increase of the energy gain (due to the formation of larger-amplitude streaks). This indicates that the p-norm optimization is well adapted to compute localized optimal disturbances only for sufficiently small values of the target time, at least in the case of shear flows for which the lift-up effect is strong. Concerning 
Table 1. Maximum absolute values of the disturbance velocity and vorticity components for the optimal perturbations obtained by linear 50-norm and nonlinear 1-norm optimizations with $T_{\mathrm{opt}}=20$ and $E_{0}=5 \times 10^{-7}$ (left columns), and with $E_{0}=7.5 \times 10^{-7}$ (right columns).

\begin{tabular}{lcccc}
\hline & $L O P, p=50$ & $W N L O P, p=1$ & $L O P, p=50$ & HNLOP, $p=1$ \\
\hline$E_{0}$ & $5 \times 10^{-7}$ & $5 \times 10^{-7}$ & $7.5 \times 10^{-7}$ & $7.5 \times 10^{-7}$ \\
$u$ & $5.0 \times 10^{-3}$ & $1.4 \times 10^{-3}$ & $6.1 \times 10^{-3}$ & $1.0 \times 10^{-2}$ \\
$v$ & $1.4 \times 10^{-2}$ & $1.7 \times 10^{-3}$ & $1.7 \times 10^{-2}$ & $7.0 \times 10^{-3}$ \\
$w$ & $1.1 \times 10^{-2}$ & $4.6 \times 10^{-3}$ & $1.3 \times 10^{-2}$ & $1.5 \times 10^{-2}$ \\
\hline
\end{tabular}

the influence of the domain length, optimizations performed for $T_{\text {opt }}=20$ using a double streamwise domain length (see figure 8(b)) further confirm that the elongation of the structures is not due to interactions of the vortices with their own tail. We can conclude that the basic shape of this p-norm optimal structure is almost independent of both the target time and the length of the domain, except for the increase of its streamwise length with the target time. Thus, as it could be anticipated, the p-norm optimals do not show similarities with the nonlinear ones, except for their strong localization, since the absence of nonlinear terms in the optimization loop inhibits nonlinear typical features such as the spanwise inclinations of the vortices. These differences are reflected also in the relative amplitudes of the velocity components. Tables 1 provides the maximum absolute values of the three velocity components for the 50-norm linear and the 1-norm nonlinear perturbations for $T_{\mathrm{opt}}=20$, $E_{0}=5 \times 10^{-7}$ (left columns) and $E_{0}=7.5 \times 10^{-7}$ (right columns). Remember that the linear optimal solutions are simply scaled by $E_{0}$ for comparison purpose, since the linear optimization procedure is independent of the value of the initial energy. The WNLOPs (second column) show values of $v$ and $w$ one order of magnitude lower than the ones obtained in the case of the p-norm LOP computed for the same target time. Although surprising, these lower values can be easily explained by noticing that the p-norm linear optimal is much more localized than the weakly nonlinear ones. For the HNLOP, the maximum amplitudes tend to increase (right column), due to the stronger localization of the flow structures. However, one can notice that the relative amplitudes of the velocity components are different: in the linear p-norm case $v$ and $w$ have similar values, being both larger than $u$, whereas for the nonlinear optimal $u \approx w, v$ being the smallest component. In fact, for linear optimals the streamwise component is always smaller than the other two components, since the initial disturbance is basically composed by streamwise vortices, characterized by the $v$ and $w$ velocity components. On the other hand, nonlinear optimizations always provide large values of the streamwise component, inducing defects in the streamwise base flow velocity profile (see Cherubini et al (2011) for the case of the boundary-layer and Cherubini and De Palma (2013) for the Couette flow). The influence of such differences on the transition scenarios initiated by these different rapidly growing perturbations will be analyzed in the next section.

\subsection{Transition mechanisms}

We have performed several Direct Numerical Simulations (DNSs) initialized by the optimal disturbances described in the previous section with several initial energies. In particular, the nonlinear optimal perturbations preserve the initial energy for which they have been computed, whereas the linear optimal perturbations have been rescaled with suitable values of $E(0)$. The initial energy values have been bisected in order to determine the minimum perturbation energy necessary to achieve transition to turbulence (where the rms curves obtained 
Table 2. Values of the transition energy thresholds associated with the optimal perturbations computed at the indicated target time.

\begin{tabular}{lll}
\hline Target time & NLOP, 1-norm & LOP, 50-norm \\
\hline 10 & $\approx 1.25 \times 10^{-6}$ & $\approx 1 \times 10^{-6}$ \\
20 & $\approx 5 \times 10^{-7}$ & $\approx 6.5 \times 10^{-7}$ \\
30 & $\approx 2.5 \times 10^{-7}$ & $\approx 6.5 \times 10^{-7}$ \\
40 & $\approx 2.5 \times 10^{-7}$ & $\approx 2 \times 10^{-6}$ \\
50 & $\approx 1 \quad \times 10^{-7}$ & $\gg 1 \times 10^{-5}$ \\
\hline
\end{tabular}

for different energy levels have been compared in order to detect transition to turbulence). These transition thresholds are provided in table 2 for the nonlinear and linear p-norm optimal perturbations. For linear 1-norm optimal perturbations, which are streamwise-independent, it is customary to add a small-amplitude noise field to the initial optimal perturbation for breaking its streamwise invariance and allow the flow to reach turbulence (Kreiss et al 1994, Reddy et al 1998). Even though this noise field is not necessary for triggering transition when initializing with the streamwise-dependent p-norm optimal disturbances (or the nonlinear ones), the transition thresholds might still be sensitive to the presence of additional random disturbances (Cossu et al 2011). Thus, we have performed further DNSs in presence of a noise field of initial energy equal to $1 \%$ and $2 \%$ of $E_{0}$, verifying that the transition thresholds provided in table 2 are marginally affected by the presence of the noise. One can see that, except for the lowest target time, $T_{\mathrm{opt}}=10$, the nonlinear optimal perturbations are able to induce transition at a lower energy than the p-norm ones. In particular, for $T_{\mathrm{opt}} \leqslant 20$, the nonlinear solutions found at the threshold energy are of the family of weakly nonlinear optimal solutions, whereas highly nonlinear optimals are found at threshold for $T_{\mathrm{opt}} \geqslant 30$. Thus, in some cases even the weakly nonlinear optimal solutions are more efficient then p-norm ones, despite their weaker localization. This confirms that, not the localization, but rather the peculiar shape of the perturbation selected by nonlinear mechanisms is crucial to efficiently induce transition.

In the following, we will discuss and compare the route to transition of linear $p$-norm and nonlinear 1-norm perturbations at the threshold energy, first in the case of short target times $\left(T_{\mathrm{opt}} \leqslant 20\right.$, discussed in section 3.2.1) and then for longer target times $\left(T_{\mathrm{opt}}>20\right.$, section 3.2.2). For characterizing and comparing the transition scenarios we have computed the rms value of the components of velocity and vorticity during the time evolution of the perturbations, the rms value of the generic variable $f$ being defined as $f_{\mathrm{rms}}^{2}=\frac{1}{V} \int_{V}(f-F)^{2} \mathrm{~d} V$, where $F$ is the value of the variable for the base flow. Moreover, Fourier transforms in $x$ and $z$ of the perturbation field have been performed on a $y=$ constant plane, in order to track the development of different modes characterized by streamwise and spanwise wavenumbers $n \alpha$ and $m \beta$, with $\alpha=2 \pi / L_{x}$ and $\beta=2 \pi / L_{z}$. In the following, the different modes will be indicated by the pair $(n, m)$ (see Schmid and Henningson (2001)).

3.2.1. Short target time analysis. In this section the transition scenarios induced by 1-norm WNLOPs and p-norm LOPs computed for $T_{\mathrm{opt}} \leqslant 20$ are analyzed and compared. Figure 9 shows the rms values of the velocity and vorticity components for the WNLOPs obtained with $T_{\text {opt }}=10, E_{0}=1.25 \times 10^{-6}$ (a)-(b) and $E_{0}=1.0 \times 10^{-6}$ (c)-(d), as well as for the 50norm LOP with $E_{0}=1.0 \times 10^{-6}(\mathrm{e})$-(f). For the WNLOP, all of the velocity components initially grow due to the Orr-mechanism for $t<10$. When the perturbation reaches a positive inclination with respect to the base flow, a modified lift-up effect (Cherubini et al (2011)) 


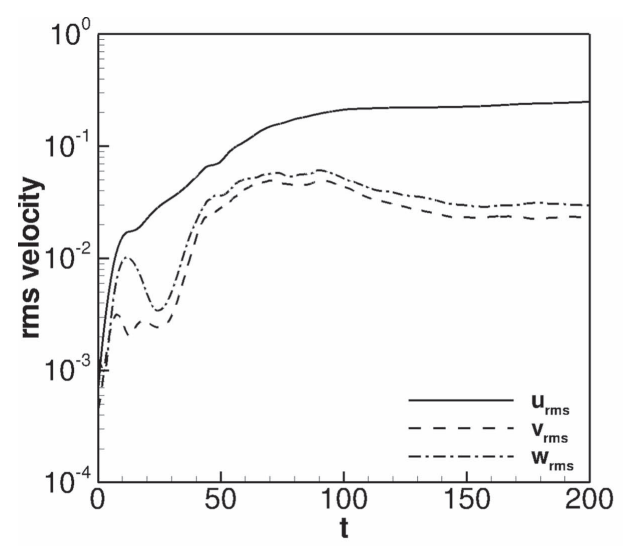

(a) WNLOP, $E_{0}=1.25 \times 10^{-6}$

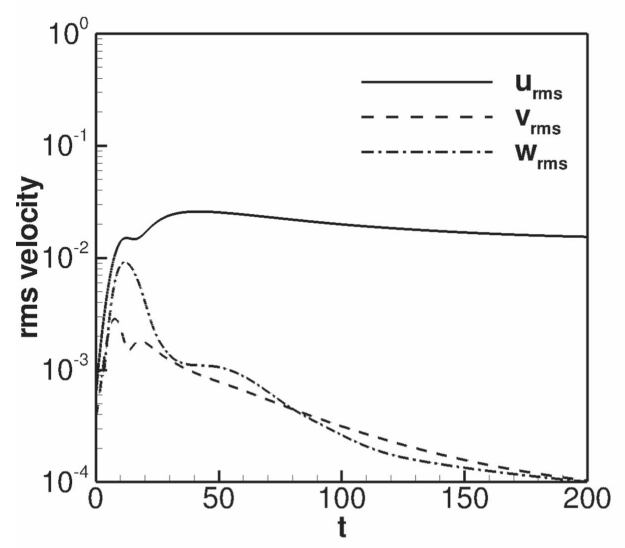

(c) WNLOP, $E_{0}=1 \times 10^{-6}$

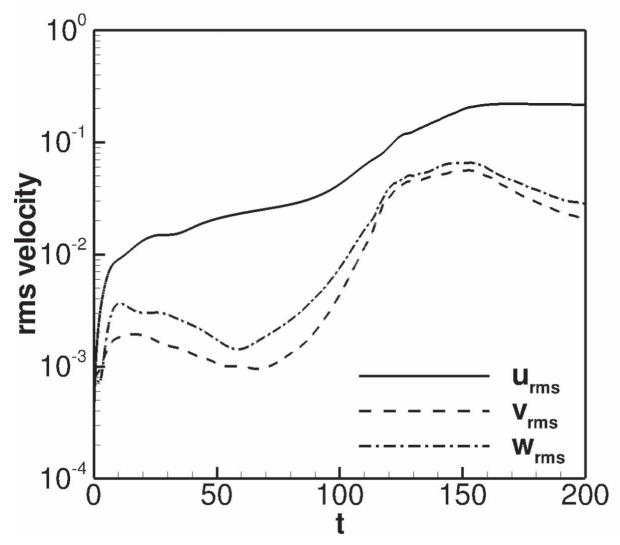

(e) 50-norm LOP, $E_{0}=1 \times 10^{-6}$

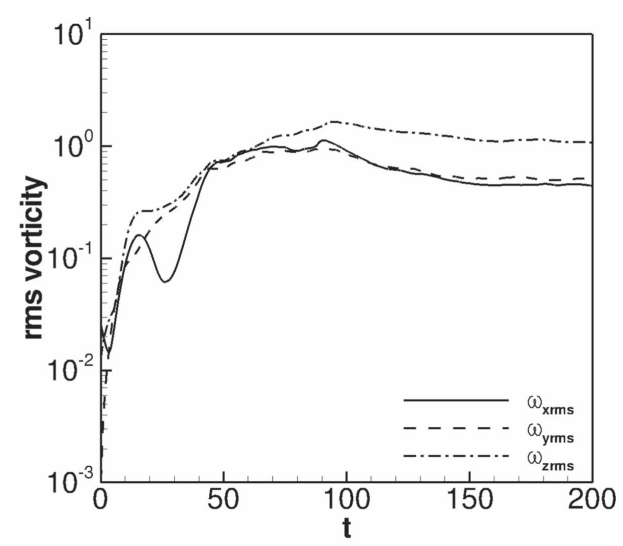

(b) WNLOP, $E_{0}=1.25 \times 10^{-6}$

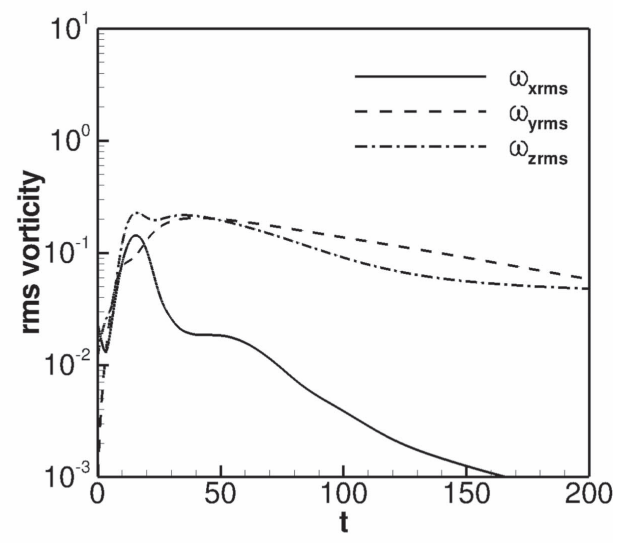

(d) WNLOP, $E_{0}=1 \times 10^{-6}$

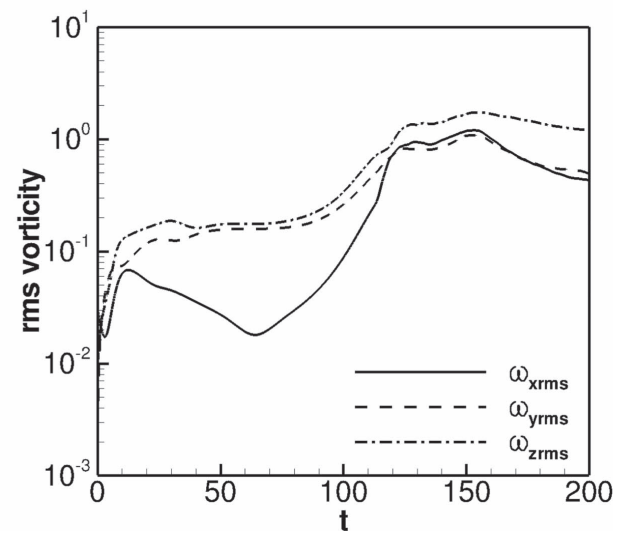

(f) 50-norm LOP, $E_{0}=1 \times 10^{-6}$

Figure 9. Time evolution of the rms of the velocity (left) and vorticity (right) components for the WNLOP with $E_{0}=1.25 \times 10^{-6}$ (top frames) and $E_{0}=1 \times 10^{-6}$ (middle frames), and of the 50-norm LOP with $E_{0}=10^{-6}$ (bottom frames), computed for $T_{\mathrm{opt}}=10$. 


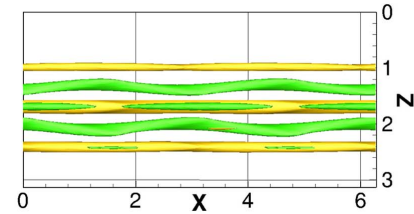

(a) $t=25$

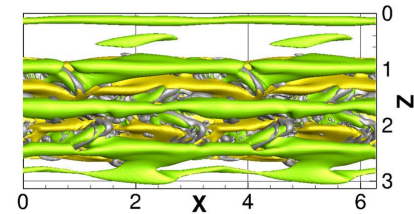

(d) $t=43$

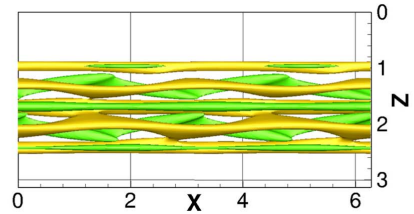

(b) $t=31$

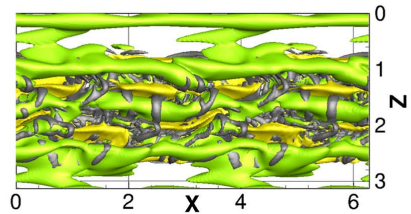

(e) $\mathrm{t}=46$

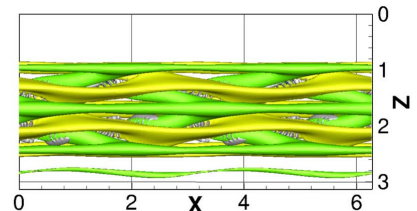

(c) $t=37$

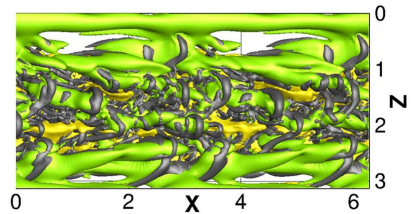

(f) $t=49$

Figure 10. Snapshots of the time evolution of the weakly nonlinear optimal perturbation found for $E_{0}=1.25 \times 10^{-6}$. Isosurface of Q-criterion $(Q=0.5$, grey), and of the streamwise velocity disturbance $(u= \pm 0.1$, green for negative and yellow for positive).

begins to induce the growth of the streamwise velocity component, while the amplitude of the $v$ and $w$ components drops (see figure 9(a) for $10<t<25$ ). Concerning the vorticity, figure 9(b) shows that after a small initial reduction of the streamwise component due to the initial tilting of the counter-rotating vortices $(t<5)$, all of the vorticity components start to grow up to $t \approx 20$. The spanwise and wall-normal components grow due to the velocity gradients induced by the formation of positive and negative streaks. Whereas, concerning the streamwise vorticity, its growth is not linked to the formation of the streaks, but rather to their modulation (Cherubini and De Palma (2013)). In particular, looking at the vorticity transport equation, for the streamwise component of the vorticity one has:

$$
\frac{D \omega_{x}}{D t}=\frac{\partial u}{\partial x} \omega_{x}+\frac{\partial u}{\partial y} \omega_{y}+\frac{\partial u}{\partial z} \omega_{z}+\frac{1}{R e} \nabla^{2} \omega_{x}
$$

At $t=0$, the wall-normal component of the vorticity is very small (see figure $9(\mathrm{~b})$ ) so the growth of $\omega_{x}$ is mostly driven by the streamwise and spanwise modulation of the streamwise velocity component. Thus, in order to allow the growth of the streamwise vorticity already at short time and rapidly trigger a self-sustained process (see Waleffe (1997)), this nonlinear optimal is characterized by a finite-amplitude streamwise velocity component modulated in $x$ and $z$. Moreover, the initial vortices are not streamwise-aligned, but they present a spanwise inclination, namely a spanwise vorticity component, at initial time. However, these mechanisms of initial growth of the streamwise velocity and vorticity are not sufficient to induce transition to turbulence, until the third element of the self-sustained process, namely the secondary instability of the streaks, is activated (Brandt et al 2000). This happens at $t \approx$ 25 , when the spanwise and wall-normal components of the velocity start to grow again in time, finally inducing transition to turbulence (at $t \approx 50$ ). If the saturated streaks are not large enough for triggering secondary instability, the flow relaminarizes after the first transient growth phases. This is clearly shown in figures $9(\mathrm{c})$ and (d), providing the velocity and vorticity rms values for the nonlinear optimal at lower initial energy, $E_{0}=10^{-6}$. These rms curves show that a perturbation having a similar shape but lower amplitude experiences a very similar evolution up to $t \approx 20$, but secondary instability is not triggered and the flow relaminarizes. 


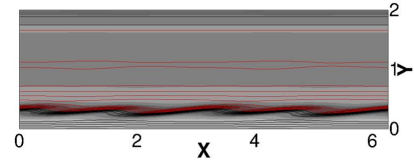

(a) $t=29$

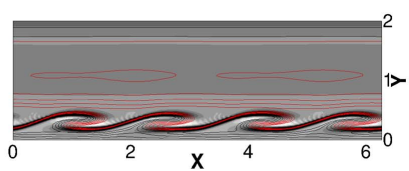

(b) $t=37$

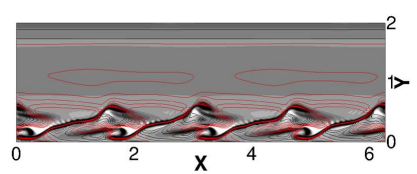

(c) $t=41$

Figure 11. Snapshots of the time evolution of the weakly nonlinear optimal perturbation computed for $E_{0}=1.25 \times 10^{-6}, T_{\mathrm{opt}}=10$. Isolines of streamwise velocity disturbance (red positive, black negative), and contours of spanwise vorticity (white positive, black negative) on a $z=2$ plane.

Snapshots of the time evolution of the weakly nonlinear optimal perturbation leading to turbulence are shown in figure 10, providing the isosurfaces of the $Q$ criterion and of the streamwise velocity disturbance. One can observe the formation of oscillating streaks at $t=25$, their growth and saturation at $t=31$ and $t=37$, followed by the development of strong vortical structures due to streak secondary instability at $t=43$ and $t=49$. The streak instability is of inflectional type (Schmid and Henningson (2001)), due to the deformation of the velocity profile in the spanwise and wall-normal direction induced by the large-amplitude negative streaks. This instability increases the spanwise and wall-normal components of the vorticity, leading to the formation of arch vortices connecting the initial neighboring vortex structures, finally forming a sequence of hairpin vortices. This mechanism can be better observed in figure 11, where the streaks (solid lines) and the spanwise vorticity (contours) are shown in a longitudinal ( $z=$ constant) plane. The creation of these large-amplitude spanwise vortices provides, through nonlinear coupling, the last element for the activation of the Waleffe self-sustaining process, eventually leading the flow to a chaotic state. We can observe three main differences with respect to the classical scenario of secondary instability of streamwise streaks (Brandt et al 2000): (i) due to the Orr mechanism, all of the velocity components initially grow; (ii) due to the initial modulation of the streamwise component of velocity, the streamwise vorticity increases too; (iii) due to the initial spanwise inclination of the streamwise vortices, the streaks are already modulated in the streamwise direction before secondary instability is triggered. Indeed, this transition scenario appears more similar to the one induced by oblique waves, which have been found to trigger transition at lower energies than streamwise vortices in shear flows (Reddy et al 1998). In this scenario, initial perturbations modulated in $x$ and $z$ are able to create, by nonlinear coupling, streamwise streaks that experience secondary instability. For analyzing this type of transition scenario, Reddy et al (1998) fed the flow by a pair of oblique waves with wavenumber $(1, \pm 1)$. These waves develop by generating streamwise vortices having wavenumber $(0,2)$, which, while decreasing in amplitude, generate streaks that saturate and experience secondary instability, inducing at later times the growth of $(2,2)$ and $(1,1)$ modes. In order to verify whether the mechanism of transition of the WNLOP shares some features with the oblique transition scenario, we analyze the evolution in time of the most amplified Fourier modes for the WNLOP, provided in figure 12 (a). One can observe that the oblique mode $(2,2)$ is indeed the mode of largest amplitude at $t=0$, indicating that the initial optimal perturbation has a modal composition similar to an oblique wave. At early times, a large growth of the streaky $(0,4)$ mode is also observed. However, these streaks do not experience a saturation phase, but they quickly break down. Moreover, many other modes grow at early times, included the initial oblique mode $(2,2)$ which does not decrease in amplitude before transition. This simultaneous, rapid growth of several modes is due to the fact that, due to the presence of nonlinear terms in the optimization procedure, this optimal perturbation has already in its 


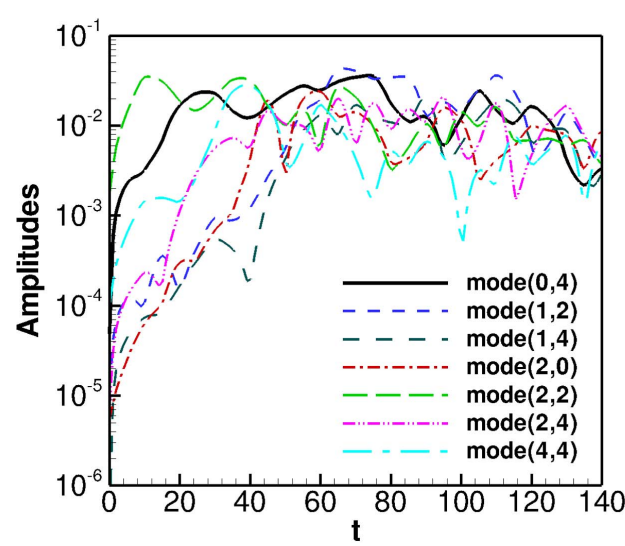

(a) Weakly nonlinear optimal

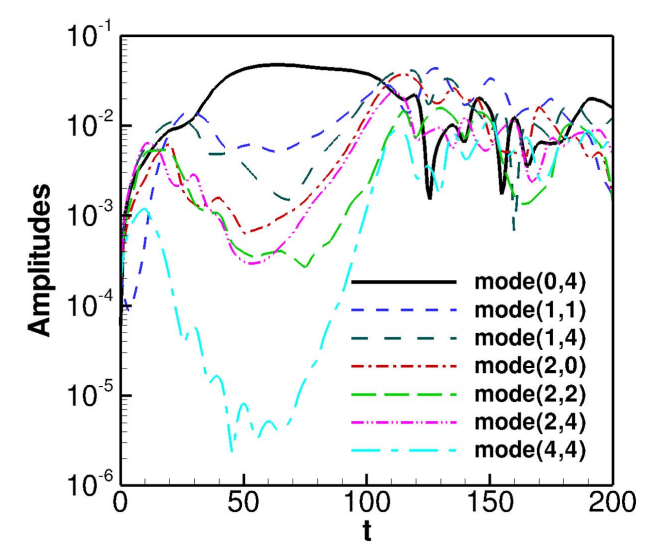

(b) Linear 50-norm optimal

Figure 12. Time evolution of the Fourier streamwise and spanwise wavenumbers for the two optimals computed at $T_{\mathrm{opt}}=10$ with initial energy $E_{0}=1.25 \times 10^{-6}$; a nonlinear 1-norm (a) and a linear 50-norm optimal (b).

initial structure the basic elements to trigger the three mechanisms of the Waleffe self sustained cycle: (i) streamwise vorticity to create the streaks; (ii) spanwise inclination to modulate them; (iii) sufficiently high amplitudes to induce nonlinear coupling.

This transition scenario for the WNLOP at $T_{\text {opt }}=10$ is now compared with the one induced by a p-norm LOP computed at the same target time, initialized with $E_{0}=10^{-6}$. Comparing figures 9 (a) and (e), one can observe that for the 50-norm case the rms-values saturate later in time (for $t>150$ instead of $t>75$ ), meaning that its evolution towards transition needs more time than the previous case. The initial growth of the velocity components due to the Orr mechanism occurs on the same time scale, but the decrease and following increase of the wall-normal and spanwise velocity components are much slower than in the nonlinear case (although the optimals have been computed for the same target time). Notice also that the streamwise vorticity increases only at early times, and then decreases reaching values quite smaller than the initial one. This is clearly due to the fact that the modulation in $x$ of the streamwise velocity component is present only at short times and fades away when streamwise streaks are created, not sustaining the growth of $\omega_{x}$.

Figure 13 provides snapshots of the evolution of streaks (yellow and green) and vortices (gray) showing the creation of bent streaks and their secondary instability. The streamwise modulation of the streaks is much weaker than in the nonlinear case (compare with figure 10), and the streaks remain almost streamwise-aligned up to $t \approx 80$, whereas in the weakly nonlinear case strong modulations are observed already in the very early phases of transition. Strong modulations of the streaks can be observed only at $t \approx 100$, leading to the formation of $\Lambda$-shaped structures that generate a population of hairpin vortices. Similarly to what has been shown before, the secondary instability of the streaks is due to the formation of the inflection points, as one can observe in the $z=$ constant sections in figure 14. However, in the nonlinear case, these inflection points occur with a different wavelength and at different times with respect to the linear case, as one can verify by comparing figure 14 with figure 11 . The wavelengths of these structures have been analyzed by performing a Fourier analysis, provided in figure 12 (b). The figure shows that the oblique mode $(2,2)$ is again the mode of largest amplitude at $t=0$, even if at early times the difference among the amplitudes of the different Fourier modes is small. Moreover, the development of the modes strongly resembles 


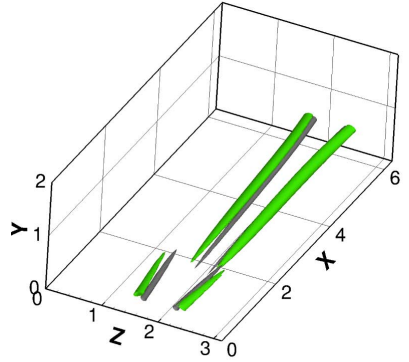

(a) $\mathrm{t}=25$

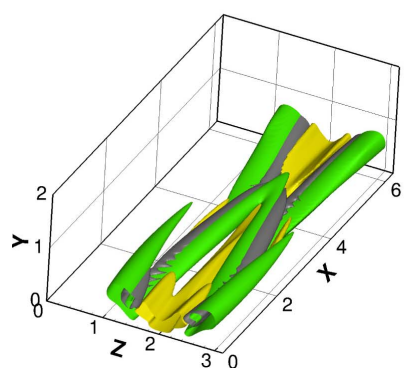

(d) $\mathrm{t}=105$

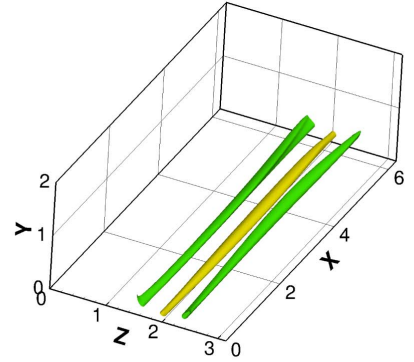

(b) $\mathrm{t}=55$

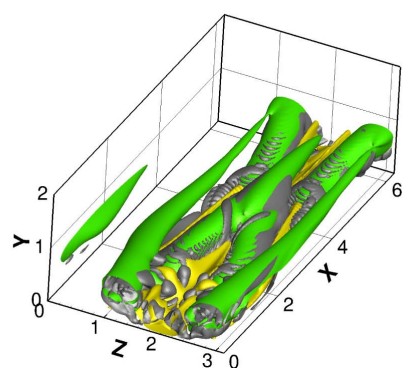

(e) $\mathrm{t}=115$

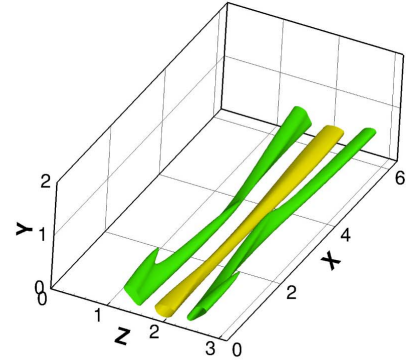

(c) $\mathrm{t}=85$

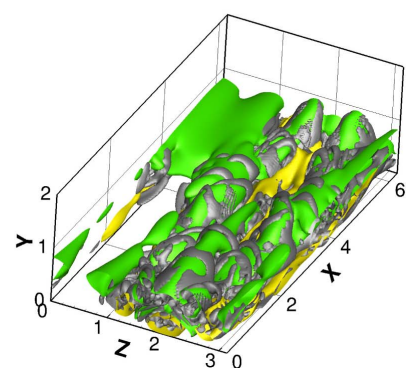

(f) $\mathrm{t}=120$

Figure 13. Evolution of linear 50-norm optimal perturbation for $T_{\mathrm{opt}}=10$ and $E_{0}=10^{-6}$. (a), (b), (c), (d), (e), (f) Isosurface of Q-criterion $(Q=0.07$, grey), and of streamwise velocity disturbance $(u=-0.9$, green and $u=0.15$, yellow).

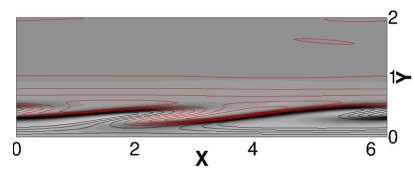

(a) $t=100$

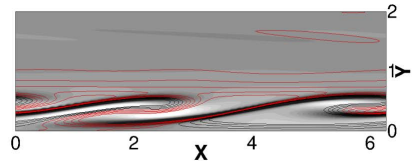

(b) $\mathrm{t}=110$

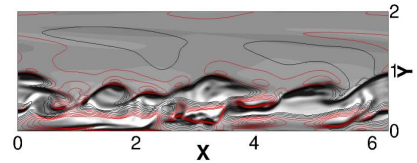

(c) $t=120$

Figure 14. Evolution of linear 50-norm optimal perturbation computed for $T_{\mathrm{opt}}=10$ and $E_{0}=10^{-6}$. Isolines of streamwise velocity disturbance (red positive, black negative), and contours of spanwise vorticity (white positive, black negative) on a $z=1.6$ plane.

that of the oblique transition scenario: except in the early phases in which the Orr mechanism is active, the growth of the streaks is associated with a strong decrease of the oblique modes (see the evolution of the $(4,4),(2,2)$ and $(1,1)$ modes in figure $12(b))$. One can also observe the long phase of saturation of the streaks represented by the long plateau of the $(0,4)$ mode. Finally, after the saturation of the streaks a new growth of the oblique modes is observed due to the secondary instability of the streaks. In particular, the streak instability is characterized mostly by modes of streamwise wavenumber 2 instead of 4 , observed for the fundamental oblique scenario (Schmid and Henningson 2001) and for the weakly nonlinear optimal perturbation evolution. In fact, comparing figure 11 with figure 14, one can observe that the Kelvin-Helmholtz instability has a double streamwise wavelength in the latter case. Thus, in the 50-norm case the secondary instability is more likely triggered by the subharmonic varicose mode instead of the fundamental one, as for the fundamental oblique wave scenario. Except for the initial modal composition, this scenario appears more similar to the oblique 


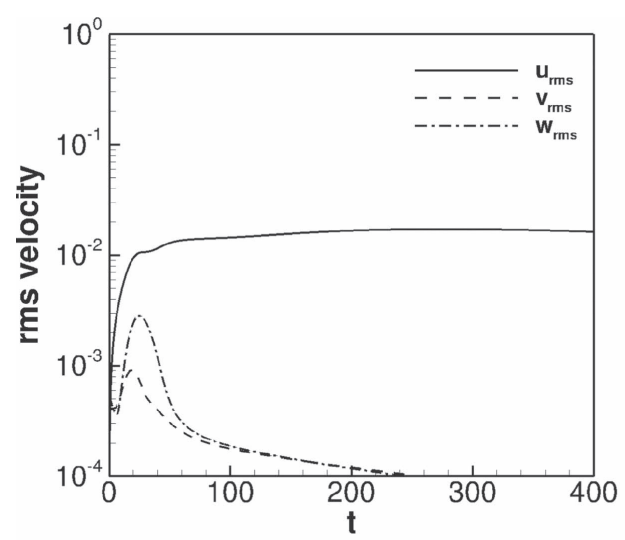

(a) 50-norm LOP

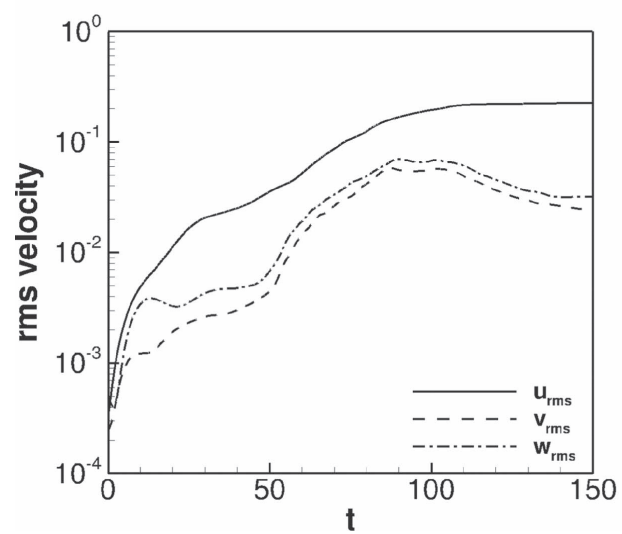

(c) HNLOP

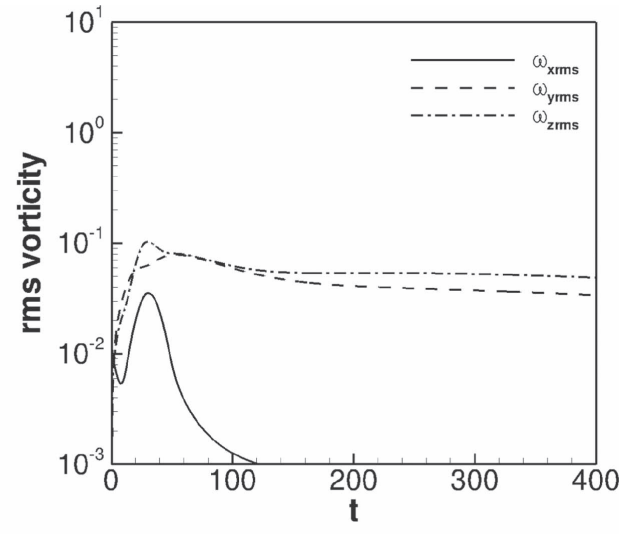

(b) 50-norm LOP

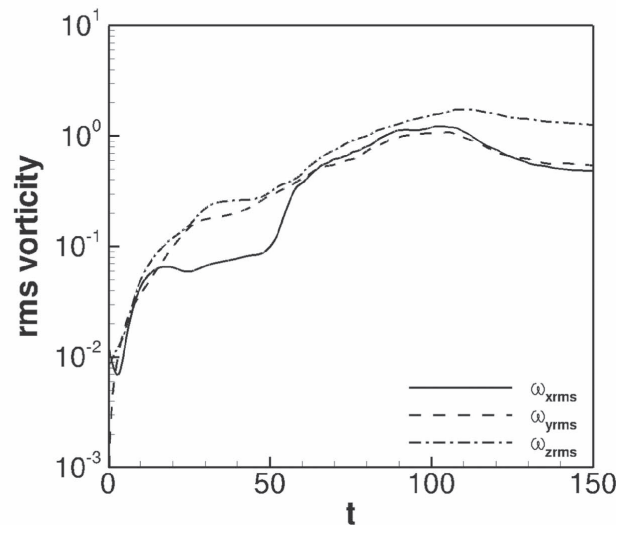

(d) HNLOP

Figure 15. Time evolution of the rms of the velocity (left) and vorticity (right) components for the 50-norm LOP (top frames) and the HNLOP (bottom frames) with $E_{0}=2.5 \times 10^{-7}$, computed for $T_{\mathrm{opt}}=30$.

scenario, being based on the generation of streamwise streaks by modulated initial perturbations, and their successive saturation and breakdown. This is due to the fact that a linear optimization can rely only on linear mechanisms to grow in time, such as the Orr and the lift-up ones, thus it has to create streaks to induce transition. On the other hand, a nonlinear optimization can couple these mechanisms by nonlinear effects to directly induce a growth of all of the modes at the same time.

Similar transition scenarios are observed for optimal perturbations computed for $T_{\text {opt }}=20$, the only difference being that the weakly nonlinear optimal experiences transition for an initial energy slightly lower than the 50-norm linear optimal (see table 2). Thus, comparing the route to transition of the WNLOP with the 50-norm LOP for $T_{\text {opt }} \leqslant 20$ can lead to two main conclusions: i) the transition route of the 50-norm optimal is similar to the subharmonic oblique transition scenario already known in the literature, whereas the WNLOP reaches transition by inducing a simultaneous growth of many different modes; ii) in terms of initial energy, the 50-norm optimal perturbation is more efficient than the weakly nonlinear 


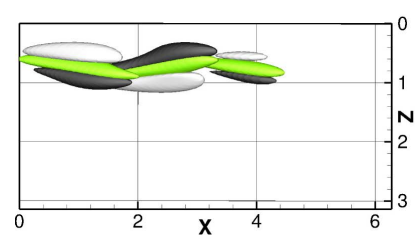

(a) $t=10$

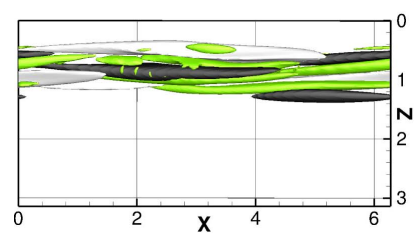

(d) $t=40$

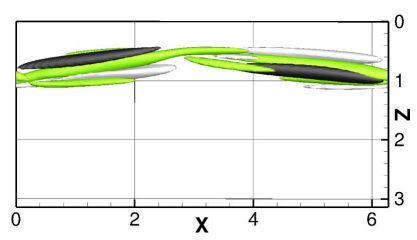

(b) $t=20$

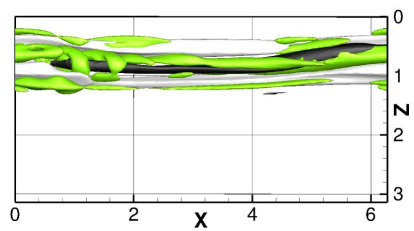

(e) $\mathrm{t}=50$

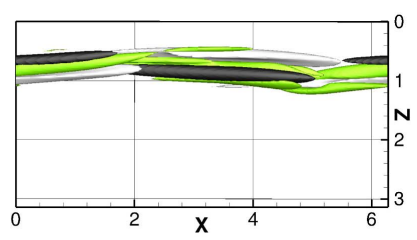

(c) $t=30$

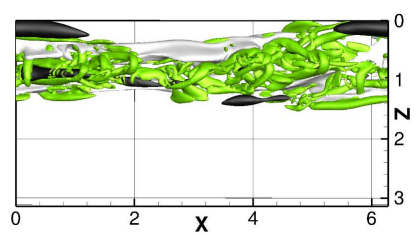

(f) $t=60$

Figure 16. Snapshots of the time evolution of the highly nonlinear optimal perturbation computed for $T_{\mathrm{opt}}=30$ and $E_{0}=2.5 \times 10^{-7}$. Isosurface of Q-criterion $(Q=0.01$ $Q_{\max }$ ) colored by streamwise vorticity, and isosurfaces of streamwise velocity disturbance $\left(u= \pm 0.5 u_{\max }\right.$, black negative and white positive).

one only at very short target time $\left(T_{\mathrm{opt}}=10\right)$, whereas it is always less efficient in terms of transition time.

3.2.2. Long target time analysis. For $T_{\mathrm{opt}}=30$, the highly nonlinear optimal is the lowestenergy solution able to lead to transition. Figure 15 shows the rms values of velocity and vorticity versus time for the 50-norm (top) and highly nonlinear (bottom) optimal perturbations computed with $E_{0}=2.5 \times 10^{-7}$ for $T_{\mathrm{opt}}=30$. The former perturbation creates streaks that saturate and then decay, since they are not accompanied by the increase of the other velocity components (except for the first slight increase due to the Orr mechanism). This is due to the fact that, for $T_{\text {opt }}>20$, the lift-up dominates the energy growth mechanisms in a linear framework, thus the perturbation is optimized with respect to a linear mechanism that cannot self-sustain in the absence of other source of growth for the $v$ and $w$ components. On the other hand, in the highly nonlinear optimal case shown in figure 15 (bottom), although a slight decrease of the $v$ component is observed after the Orr mechanism, these components are found to grow together with the streamwise component. Moreover, similarly to what has been observed in figure 9 for a lower $T_{\text {opt }}$ and a larger $E_{0}$, the streamwise component does not need to saturate to induce the $v$ and $w$ increase, since the three components of velocity are nonlinearly coupled. One can also observe that, although a lower initial energy is imposed with respect to the WNLOP case, transition is reached very quickly, since the highly nonlinear optimal found for these values of the energy and target time exploits all of the nonlinear effects to rapidly induce transition.

Snapshots of the evolution towards transition for the HNLOP computed with $T_{\mathrm{opt}}=30$ and $E_{0}=2.5 \times 10^{-7}$ are shown in figure 16. After tilting downstream exploiting the Orr mechanism (first frame for $t=10$ ), the inclined vortices begin to transport the base-flow momentum, creating localized and modulated zones of high and low streamwise perturbation (black and white isosurface of streamwise velocity disturbance). These positive and negative zones are initially alternated in $x$ and $z$, and the initial vortices are localized in space. As time increases, the vortices are stretched in the streamwise directions by the mean flow (compare the second and third frame for $t=20$ and $t=30$ ); as a consequence, at $t=40$ the 


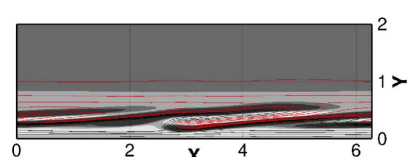

(a) $t=40$

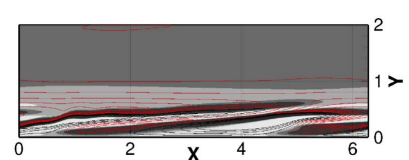

(b) $t=50$

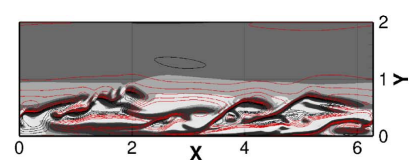

(c) $t=60$

Figure 17. Snapshots of the time evolution of the HNLOP computed for $E_{0}=2.5 \times 10^{-7}, T_{\mathrm{opt}}=30$. Isolines of streamwise velocity disturbance (red positive, black negative), and contours of spanwise vorticity (white positive, black negative) on a $z=2$ plane.

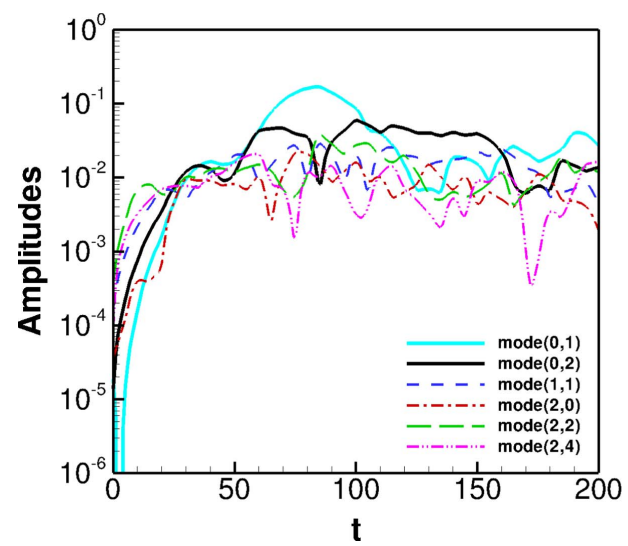

(a) Highly nonlinear optimal

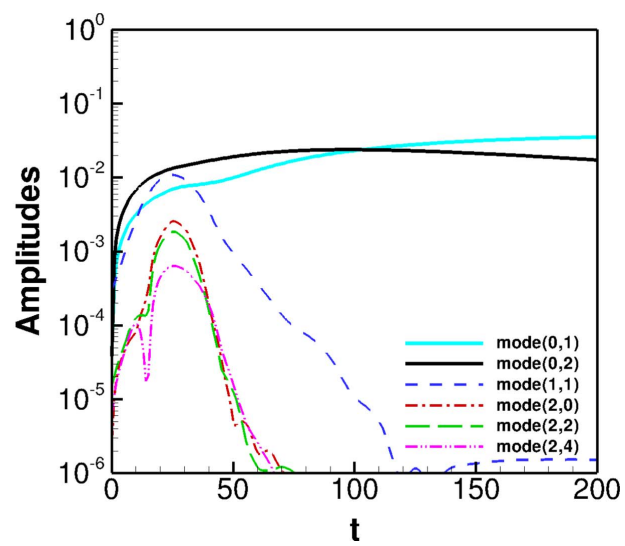

(b) Linear 50-norm optimal

Figure 18. Time evolution of the Fourier streamwise and spanwise wavenumbers for the two optimals computed at $T_{\text {opt }}=30$ with initial energy $E_{0}=2.5 \times 10^{-7}$; a nonlinear 1-norm (a) and a linear 50-norm optimal (b).

nonsymmetric vortices begin to merge on top of the negative streamwise velocity disturbance, creating cane-like vortical structures. At the same time, the regions of positive and negative streamwise velocity begin to merge, inducing at $t=50$ the creation of elongated bent streaks. In particular, two high-speed bent streaks can be observed on the side of a central low-speed streak; in the zone of interaction of the low- and the high-speed streaks ( $x \approx 1$ in figure 16(e)), strong vorticity is created due to the presence of strong inflection points. This local instability then produces smaller-scale vortical structures leading the flow to turbulence (sixth frame for $t=60$ ). The evolution of spanwise vorticity (isocontours) and streamwise velocity (isolines) perturbation is shown in a $z=$ const plane in figure 17. One can observe the complex alternated pattern given by the low- and high-speed streaks, and the strong vorticity created in localized regions of the flow, leading to turbulence already at $t=60$ (compare with figure 14, in which a chaotic behaviour is observed only at $t=120$ ). It is also noteworthy that the dynamics of the HNLOP is similar to the one found for nonlinear optimal disturbances in Couette flow (Cherubini and De Palma 2013), indicating that in parallel shear flows this nonlinear optimal transition scenario has a general relevance.

The Fourier transform of the velocity signal extracted each five time units, provided in figure 18(a), shows that all of the Fourier modes grow at the same time, with similar slopes and amplitudes. Moreover, no saturation phase is observed for the streaky modes, $(0,1)$ and $(0,2)$. Thus, the HNLOP is able to induce a very rapid simultaneous growth of all of the 
modes; this growth is even more rapid than the one found for the WNLOP, which could rely on weaker nonlinear effects. On the other hand, the 50-norm linear optimal computed for the same target time and normalized with the same initial energy only induces the growth of the streaky modes, $(0,1)$ and $(0,2)$, which very rapidly increase their energy, as shown in figure 18(b). However, while the streaks slowly saturate in time, the other modes rapidly decay, since nonlinear effects are not exploited enough to sustain a regeneration cycle.

In conclusion, p-norm linear and 1-norm nonlinear optimal disturbances appear to be characterized by important differences in their route to transition. Both perturbations rely only on linear energy growth mechanisms in their initial evolution phases; however, nonlinear optimal perturbations are able to exploit all of the energy growth mechanisms occurring over different timescales, allowing a simultaneous growth of different interacting modes which successfully lead the flow to transition.

\section{Conclusions}

Localized optimal perturbations (OPs) for plane Poiseuille flow have been computed by using two optimization approaches based on the Lagrange multipliers formulation: a linear optimization, using the p-norm (with $p=50$ ) of the energy perturbation as objective function, and a nonlinear optimization, considering the energy perturbation as objective function. These OPs maximize the objective function at a given target time $T_{\mathrm{opt}}$ and for a given initial energy $E_{0}$ in the case of the nonlinear procedure. The shape of the p-norm linear OP is rather robust. In fact, for all of the considered target times and initial energies, it is characterized by a pair of localized vortices aligned with the streamwise direction and mirror-symmetric with respect to the spanwise direction. A weaker localization in the streamwise direction is recovered for large target times, the streamwise vortices elongating in the streamwise direction, whereas the spanwise length of the disturbance remains unchanged. On the other hand, the use of the nonlinear approach allows one to unravel the rich structure of optimal perturbations which change strongly depending on target time and initial energy. In fact, for low values of $T_{\text {opt }}\left(T_{\text {opt }}<20\right)$ and intermediate values of $E_{0}$, a weakly nonlinear optimal perturbation has been found, composed by alternated vortices inclined with respect to the streamwise direction, with large values of the streamwise velocity disturbance. This OP localizes at one of the two walls, thus breaking the wall-normal symmetry of the linear OPs. For larger target time $\left(T_{\mathrm{opt}} \geqslant 20\right)$, a highly nonlinear optimal perturbation is found for sufficiently high values of $E_{0}$, which breaks also the spanwise symmetry. In this case, the disturbance strongly localizes in all of the spatial directions, being composed of spanwise inclined vortices along patches of large-amplitude streamwise velocity, very similar to the nonlinear OP found for Couette flow (Cherubini and De Palma 2013). Finally, for very short target times and high values of the initial energy, a hairpin-like nonlinear optimal perturbation is obtained (not analyzed in the present paper). Thus, although both linear and nonlinear optimizations provide localized OPs, the shape and characteristics of linear p-norm and nonlinear OPs are rather different: (i) p-norm optimal vortices are streamwise-aligned, whereas nonlinear ones are inclined; (ii) p-norm OPs loose their streamwise localization when the target time is increased, whereas the nonlinear OPs become more and more localized; (iii) for sufficiently high initial energies, the relative amplitudes of the velocity components differ, the streamwise component being the smallest for the linear p-norm OP, the wall-normal component, instead, for the nonlinear OP.

Concerning the route to transition, the p-norm OP follows a transition path similar to the oblique transition scenario, with slightly oscillating streaks, created by a modulated initial perturbation, which saturate and then experience secondary instability. The weakly nonlinear 
OP has an initial modal composition resembling an oblique wave. However, it can induce a faster transition by generating bent large amplitude streaks instead of streamwise aligned ones, skipping the phase of saturation typical of the oblique scenario. On the other hand, for the highly nonlinear OPs, all of the velocity components grow together, due to important nonlinear effects leading to transition very efficiently. As expected, in most of the cases the nonlinear optimals induce transition to turbulence for lower energies (about one third for $T_{\text {opt }}=30$ ) and in a shorter time than the p-norm ones. Decreasing the initial energy of a factor three can be very important in industrial applications or for actuators controlling the flow. Moreover, nonlinear optimal disturbances computed for a very long target time can provide the minimal-energy threshold for transition, allowing one to determining the amplitude thresholds for conditional stability in subcritical transition. Thus, an open and interesting question might be the physical meaning and benefits of p-norm OPs computed in a nonlinear framework. Using both nonlinearity and higher-order norms to localize the initial perturbation could provide interesting results. Future works will aim at studying these open questions.

\section{Acknowledgments}

Part of this work was performed using HPC resources from GENCI-[CCRT/CINES/IDRIS] (Grant x20142a6362).

\section{Appendix. Optimisation method}

The augmented functional for the optimisation reads:

$$
\begin{aligned}
& \mathfrak{L}\left(u_{k}, p, u_{k}^{\dagger}, p^{\dagger}, u_{k}(T), u_{k}(0), \lambda\right) \\
&= \frac{E_{p}\left(T_{\mathrm{opt}}\right)}{E(0)}-\int_{0}^{T_{\mathrm{opt}}} \int_{V} u_{i}^{\dagger}\left(\frac{\partial u_{i}}{\partial t}\right. \\
&\left.\quad+\frac{\partial\left(u_{i} U_{j}\right)}{\partial x_{j}}+\frac{\partial\left(U_{i} u_{j}\right)}{\partial x_{j}}+\frac{\partial p}{\partial x_{i}}-\frac{1}{R e} \frac{\partial^{2} u_{i}}{\partial x_{j}^{2}}+\frac{\partial\left(u_{i} u_{j}\right)}{\partial x_{j}}\right) \mathrm{d} t \mathrm{~d} V \\
& \quad-\int_{0}^{T_{\mathrm{opt}}} \int_{V} p^{\dagger} \frac{\partial u_{i}}{\partial x_{i}} \mathrm{~d} t \mathrm{~d} V-\lambda\left(\frac{E_{0}}{E(0)}-1\right) .
\end{aligned}
$$

Therefore, looking for the extrema of $\mathfrak{L}$ with respect to every independent variable, we get the equations that close the optimization problem:

$$
\begin{gathered}
\frac{\partial \mathfrak{L}}{\partial \lambda}=\frac{E_{0}}{E(0)}-1=0 \\
\frac{\partial \mathfrak{L}}{\partial u_{k}^{\dagger}}=\frac{\partial u_{k}}{\partial t}+\frac{\partial\left(u_{k} U_{j}\right)}{\partial x_{j}}+\frac{\partial\left(U_{k} u_{j}\right)}{\partial x_{j}}+\frac{\partial p}{\partial x_{k}}-\frac{1}{R e} \frac{\partial^{2} u_{k}}{\partial x_{j}^{2}}+\frac{\partial\left(u_{k} u_{j}\right)}{\partial x_{j}}=0 \\
\frac{\partial \mathfrak{L}}{\partial p^{\dagger}}=\frac{\partial u_{i}}{\partial x_{i}}=0 \\
\frac{\partial \mathfrak{L}}{\partial p}=\frac{\partial u_{i}^{\dagger}}{\partial x_{i}}=0
\end{gathered}
$$




$$
\begin{gathered}
\frac{\partial \mathfrak{L}}{\partial u_{k}}=\frac{\partial u_{k}^{\dagger}}{\partial t}+\frac{\partial\left(u_{k}^{\dagger} U_{j}\right)}{\partial x_{j}}-u_{i}^{\dagger} \frac{\partial U_{i}}{\partial x_{k}}+\frac{\partial p^{\dagger}}{\partial x_{k}}+\frac{1}{R e} \frac{\partial^{2} u_{k}^{\dagger}}{\partial x_{j}^{2}} \\
+\underbrace{\frac{\partial\left(u_{k}^{\dagger} u_{j}\right)}{\partial x_{j}}-u_{i}^{\dagger} \frac{\partial u_{i}}{\partial x_{k}}}_{\text {direct }- \text { adjoint coupling terms }}=0 \\
\frac{\partial \mathfrak{L}}{\partial u_{k}(T)}=\frac{\left(\frac{1}{V} \int_{V}\left(\frac{1}{2} u_{i}(T) u_{i}(T)\right)^{p} \mathrm{~d} V\right)^{\left(\frac{1}{p}-1\right)}\left(\frac{1}{2} u_{i}(T) u_{i}(T)\right)^{(p-1)} u_{k}(T)}{E(0)}-u_{k}^{\dagger}(T)=0 \\
\frac{\partial \mathfrak{L}}{\partial u_{k}(0)}=-\frac{E_{p}(T)-\lambda E_{0}}{E(0)^{2}} u_{k}(0)+u_{k}^{\dagger}(0)=0 .
\end{gathered}
$$

Equations (A.2)-(A.4) represent the constraints of the optimization problem. Equations (A.5) and (A.6) are the perturbative adjoint Navier-Stokes equations, obtained after integration by parts of equations (A.1) and successive derivation with respect to the direct variables. Imposing the boundary conditions on the direct variables, the following boundary conditions are obtained for the adjoint variables: $u_{i}^{\dagger}=0$ and $p^{\dagger}=0$ at $y$-constant boundaries; periodic boundary conditions at the other boundaries. Equation (A.7) represents the compatibility conditions between the direct and the adjoint problem at $t=T_{\mathrm{opt}}$, whereas equation (A.8) is the gradient that we aim at nullifying. Notice that equation (A.7) is responsible for the localization of the optimal perturbation when the p-norm of the energy density is used as the objective function. As described by Foures et al (2013), initializing the optimization process with a random noise localized in a given region of the flow, step (A.7) of the procedure will localize the perturbation more and more during the optimization process. Thus, the algorithm wouldn't have any possibility of exploring other regions of the solution space, providing the local optimal solution for that particular initialization.

These equations are solved by using an iterative procedure. The optimization cycle starts providing an initial guess for the optimal perturbation $u_{i}(0)$; then, the Navier-Stokes equations (A.3) and (A.4) (direct problem) are solved forward in time up to the target time. Then, the adjoint variables are computed by using the compatibility condition (A.7) at $\left(t=T_{\mathrm{opt}}\right)$, and the adjoint equations (A.5), (A.6) are solved backward in time up to $t=0$. To switch back to direct variables, and close the optimization loop, we use the gradient-based methods proposed by Foures et al (2013). At each direct-adjoint iteration, the objective function is evaluated in order to assess if its variation between two successive iterations, $\epsilon$, is smaller than a chosen threshold. In this case, the cycle is stopped. To keep the computational cost affordable, a threshold value equal to $\epsilon=10^{-5}$ has been chosen. Concerning the convergence of the algorithm, figure 19 shows: (i) the residual $\epsilon$, (ii) the energy gain, (iii) the normalised residual $r=\frac{\left\|\nabla \mathfrak{I}^{\mathfrak{L}}\right\|_{2}^{2}}{\|\nabla \mathfrak{L}\|_{2}^{2}}$, versus the iterations, for two different non-linear optimizations providing the hairpin-like OP (left) and the HNLOP (right), respectively. As one can see, in both cases the energy reaches an (almost) constant value, and the value of $\epsilon$ is not seen to oscillate at the end of the iteration process. Concerning the normalised residual $r$, for both optimization it reaches a value of $O\left(10^{-4}\right)$, with a decreasing smooth tendency in the first case, and an asymptotic value for the second, indicating convergence of the algorithm. It is noteworthy that the same procedure is used for a linear or nonlinear optimization, the only difference being that the nonlinear terms in equation (A.3) are set to zero in the linear case. 


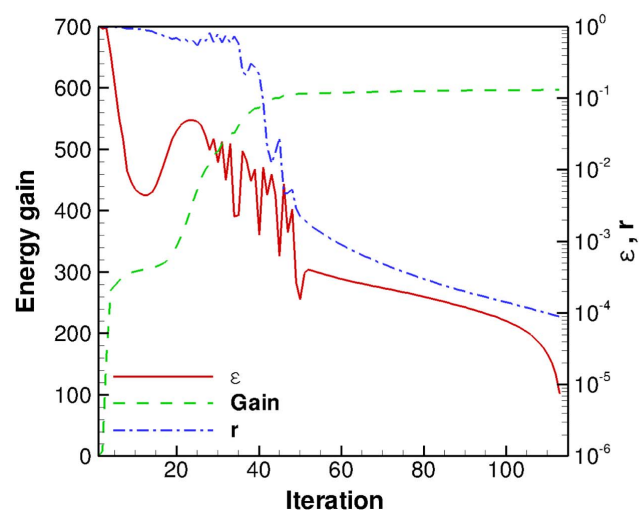

(a)

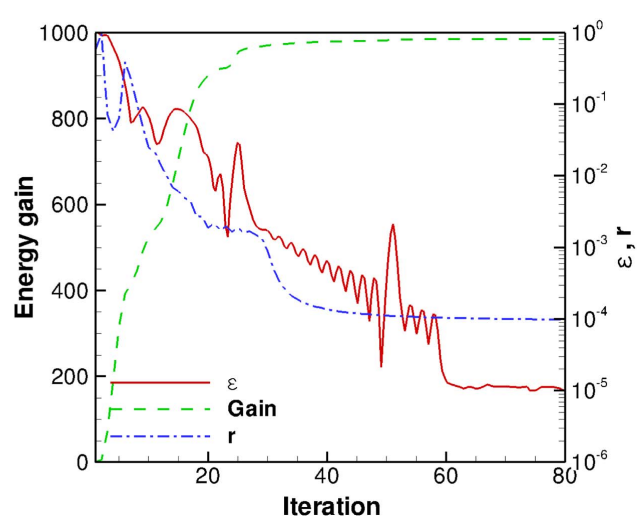

(b)

Figure 19. Energy gain (green dashed line) $\epsilon$ (blue dash-dotted line) and $r$ (red solid line) plotted against iteration for (left) the Hairpin-like OP, $T_{\text {opt }}=16$ at $E_{0}=2 \times 10^{-6}$ and (right) the HNLOP $T_{\text {opt }}=30$ at $E_{0}=2.5 \times 10^{-7}$.

This implies the cancellation of the direct-adjoint coupling terms in equation (A.6). The absence of these terms makes the procedure much faster, since for evaluating these coupling terms the direct variables need to be stored at each time step of the direct problem integration in order to be available for the integration of the adjoint equations backward in time.

\section{References}

Avila K, Moxey D, de Lozar A, Avila M, Barkley D and Hof B 2011 Science 333 192-6

Bottin S and Chaté H 1998 Eur. Phys. J. B 6 143-55

Brandt L, Andersson P and Henningson D 2000 Advances in Turbulence VIII Proc 8th European

Turbulence Conference (Barcelona, 27-30 June) pp 141-4

Butler K M and Farrell B F 1992 Phys. Fluids A 4 1637-50

Cherubini S and De Palma P 2013 J. Fluid Mech. 716 251-79

Cherubini S, De Palma P, Robinet J and Bottaro A 2011 J. Fluid Mech. 689 221-53

Cherubini S, De Palma P and Robinet J C 2015 Phys. Fluids 27034108

Cherubini S, De Palma P, Robinet J C and Bottaro A 2010 Phys. Rev. E 82066302

Cherubini S, Robinet J, Bottaro A and Da Palma P 2010 J. Fluid Mech. 656 231-59

Cherubini S, Robinet J C and De Palma P 2013 J. Fluid Mech. 737 440-65

Cossu C, Brandt L, Bagheri S and Henningson D S 2011 Phys. Fluids 23074103

Darbyshire A and Mullin T 1995 J. Fluid Mech. 289 83-114

Dauchot O and Daviaud F 1995 Phys. Fluids 7 335-43

Duguet Y, Monokrousos A, Brandt L and Henningson D S 2013 Phys. Fluids 25084103

Farano M, Cherubini S, Robinet J C and De Palma P 2015 J. Fluid Mech. 775 R2

Farrell B F 1988 Phys. Fluids 312093

Foures D, Caulfield C and Schmid P 2013 J. Fluid Mech. 729 672-701

Foures D, Caulfield C and Schmid P J 2012 Phys. Rev. E 86026306

Henningson D S, Lundbladh A and Johansson A V 1993 J. Fluid Mech. 250 169-207

Hinze M, Pinnau R, Ulbrich M and Ulbrich S 2009 Optimization with PDE Constraints of Mathematical Modelling: Theory and Applications vol 23 (Berlin: Springer)

Hof B, van Doorne C W, Westerweel J and Nieuwstadt F T 2005 Phys. Rev. Lett. 95214502

Hunt J C R, Wray A and Moin P 1988 Center for Turbulence Research Report Report N89-24555

Kreiss G, Lundbladh A and Henningson D S 1994 J. Fluid Mech. 270 175-98

Landahl M 1980 J. Fluid Mech. 98 243-51

Levin O and Henningson D S 2007 J. Fluid Mech. 584 397-413 
Mellibovsky F and Meseguer A 2007 Phys. Fluids 19044102

Monokrousos A, Akervik E, Brandt L and Henningson D S 2010 J. Fluid Mech. 650 181-214

Monokrousos A, Bottaro A, Brandt L, Di Vita A and Henningson D 2011 Phys. Rev. Lett. 106134502

Patel V and Head M 1969 J. Fluid Mech. 38 181-201

Perry A, Lim T and Teh E 1981 J. Fluid Mech. 104 387-405

Pringle C C T and Kerswell R 2010 Phys. Rev. Lett. 105154502

Pringle C C, Willis A P and Kerswell R R 2012 J. Fluid Mech. 702 415-43

Rabin S, Caulfield C and Kerswell R 2012 J. Fluid Mech. 712 244-72

Reddy S C, Schmid P J, Baggett J S and Henningson D S 1998 J. Fluid Mech. 365 269-303

Reynolds O 1883 Proc. of the Royal Society of London 35 84-99

Schlichting H and Gersten K 2000 Boundary-Layer Theory (Berlin: Springer)

Schmid P J and Henningson D S 2001 Stability and Transition in Shear Flows vol 142 (Berlin: Springer)

Singer B A 1996 Phys. Fluids 8 509-21

Singer B A and Joslin R D 1994 Phys. Fluids 6 3724-36

Suponitsky V, Cohen J and Bar-Yoseph P 2005 J. Fluid Mech. 535 65-100

Trefethen L et al 1993 Science 261 578-84

Verzicco R and Orlandi P 1996 J. Comput. Phys. 123 402-14

Waleffe F 1997 Phys. Fluids 9 883-900

Wygnanski I and Champagne F 1973 J. Fluid Mech. 59 281-335

Wygnanski I, Sokolov M and Friedman D 1976 J. Fluid Mech. 78 785-819

Zuccher S, Luchini P and Bottaro A 2004 J. Fluid Mech. 513 135-60 